\title{
Coupled analysis of integrated dynamic responses of side-by-side offloading FLNG system
}

\author{
Dongya Zhao ${ }^{\mathrm{a}, \mathrm{c}}$, Zhiqiang $\mathrm{Hu}^{\mathrm{b} *}$, Ke Zhou ${ }^{\mathrm{a}, \mathrm{c}}$, Gang Chen ${ }^{\mathrm{a}, \mathrm{d}}$, Xiaobo Chen ${ }^{\mathrm{e}}$, Xingya Feng \\ a State Key Laboratory of Ocean Engineering, Shanghai Jiao Tong University, 800 Dongchuan Road, Shanghai, China \\ b School of Engineering, Newcastle University, Newcastle upon Tyne, NE1 7RU, UK \\ ${ }^{c}$ Collaborative Innovation Center for Advanced Ship and Deep-Sea Exploration, Shanghai Jiao Tong University, Shanghai, China \\ ${ }^{\mathrm{d}}$ Marine Design \& Research Institute of China, Shanghai, China \\ e Deepwater Technology Research Centre, Bureau Veritas, Singapore
}

\begin{abstract}
Integrated dynamic responses of FLNG system in side-by-side offloading operation are investigated numerically and experimentally in this paper. A numerical code is developed based on potential flow theory to predict the interactions between connected vessels' motions and liquid sloshing in the time domain. The impulsive response function (IRF) method is adopted in the resolution for 6 DOF vessels' motions, nonlinear sloshing in liquid tanks is solved using boundary element method (BEM), and connection system including hawsers and fenders is numerically modeled as linear response system. A series of model tests are conducted to validate the feasibility of the numerical code. Hydrodynamic interaction between the vessels and shielding effects under different wave directions are analyzed; sloshing effects on the vessels' motions and on the loads of connection system are investigated; the sensitivities of the vessels' motions and loads to connection system stiffness are discussed. It is found that the vessels' motions are significantly affected by the hydrodynamic interactions between vessels and sensitive to wave directions for shielding effects. The radiation forces of the adjacent vessel tend to amplify vessel's motions and LNG carrier is more likely to be affected by FLNG's radiation forces for their difference in displacement. In addition, compared with solid loading condition, liquid loading vessel tends to have decreased natural roll frequency and have increased sway motions in the frequency region that higher than the natural sloshing frequency. The two sloshing responses peaks appear in the natural roll frequencies and natural sloshing frequencies, which are respectively mainly excited by vessels' roll and sway motions. Besides, in low fill conditions, the sloshing loads contribute to larger sway motions in low fill conditions for the natural sloshing frequencies are closer to the main response frequency region of vessels. Sloshing nonlinearity gets obvious in the conditions with low fill conditions and large wave amplitude, while the motion responses of vessels have slight nonlinearity with the increases of sloshing nonlinearity when no violent sloshing with wave break is excited. Furthermore, small stiffness of the connection system has slight influences on the vessels' motions, and resonant motions can be excited when the natural frequency of the connection system located in the wave frequency region.
\end{abstract}

Keywords: FLNG system; coupling interaction; sloshing; potential flow theory; model test

\section{Introduction}

The development of floating liquefied natural gas (FLNG) system that can produce, process and store liquefied natural gas (LNG) offshore has been stimulated by the increasing demand of natural gas. One challenge for the operation of FLNG system is LNG offloading to an LNG carrier. Considering the efficiency and the limitation of flexible pipeline in transferring LNG under extremely low temperature, side-by-side configuration is more economically attractive than tandem configuration in offloading operation. In the hydrodynamic perspective, the side-by-side arrangement of a FLNG system involves interactions between multiple bodies, sloshing in liquid tanks and resonance in the narrow gap between vessels. Besides, the connection system adopted to restrict the 
relative motions between vessels also plays a crucial role, and attentions should also be paid to the loads on the connection system.

Hydrodynamic interactions among floating bodies in proximity exist in many applications in ocean engineering and have received many researchers' attentions. Kodan (1984) analyzed two parallel floating structures using strip theory. Inoue \& Islam (1999) calculated motions of multiple floating offshore structures with consideration of the effects of connectors and mooring lines in the frequency domain. Buchner et al. (2001) developed a numerical model to predict the hydrodynamic responses of paralleled moored vessels in the time domain, where hydrodynamic coefficients were prepared in the frequency domain. Newman (2001) presented numerical and analytical results in the research of multiple floating bodies, first and second order interaction effects were considered. Choi \& Hong (2002) and Hong et al. (2005) applied high order boundary element method (HOBEM) to simulate side-by-side vessels in the time domain. To research the coupling mechanism of two adjacent vessels, Koo \& Kim (2005) compared three different numerical methods in combining motion equations. Zhu et al. (2008) developed a numerical program to simulate hydrodynamic interactions of three-dimensional multiple floating structures in the time domain based on potential flow theory, the governing equations were solved in time domain directly rather than using IRF. Pessoa et al. (2015) conduced numerical and experimental research on two floating structures connected with mooring lines and fenders. First order relative motions and loads on connection system were investigated. Zhao et al. (2012) carried out numerical simulation of FLNG system in parallel arrangement with time-domain analysis code SIMO. Xu et al. (2015) studied coupled motions of three barges in side-by-side arrangement; a numerical program was developed and validated by test results. Based on relative motions between vessels, loads on connection system were obtained by assuming the connection system reacts quasi-statically (Hong et al., 2005; Zhao et al., 2014; Pessoa et al., 2015).

For floating vessels in proximity, resonance in the gap between vessels is also a complicated phenomenon and is of practical importance. Molin (2001) derived analytical solution of resonance in moon pools. Sun et al. (2010) studied the first and second order resonant properties between barges in parallel. The results of model tests showed that resonant frequencies would shift depending on whether the barges were fixed or floating. Zhao et al. (2017 a) experimentally investigated the resonant sloshing responses in the gap between two fixed barges. In the calculation of hydrodynamic coefficients in the frequency domain, potential flow theory tends to over-predict resonant responses in the gap and generates inaccurate hydrodynamic coefficients for no viscosity was accounted. Huijsmans et al. (2001) applied a rigid lid in the gap to suppress the unrealistic prediction. Chen (2005) modified the free surface condition between vessels by introducing an artificial damping term to suppress resonant fluid in the gap. This method had been used in sloshing problems to account for energy dissipation (Faltinsen, 1978). Lu et al. (2011) and Yao \& Dong (2015) also applied a dissipating parameter to study the wave elevation in the gap, and sensitivities to the parameter value were discussed. It was found that the artificial damping term can suppress unrealistic resonant sloshing effectively, but the selection of proper value still needs much effort.

Moreover, sloshing in the liquid tanks in FLNG system makes vessels' motion responses much different from solid loading condition. To date, many methods have been adopted in the predication of sloshing in liquid loading vessel. Lee et al. (2007) and Nam et al. (2009) calculated sloshing in a liquid tank through a Navier-Stokes solver, and coupled the sloshing loads with the ship motion solved by linear potential flow. Jiang et al. (2016) used the open source computational fluid dynamics (CFD) code OpenFOAM to investigate the couplings in liquid loading vessels in the time domain and found impact loads due to violent sloshing had less effect on ship's global motion responses. CFD methods solving Navier-Stokes equations tend to have low efficiency in dealing with FLNG system with large liquid tanks and various fill conditions. Malenica et al. (2003) considered dynamic coupling of liquid loading vessel in the frequency domain, and linear potential theory was adopted in the solving both sloshing and vessel's seakeeping properties. Rognebakke \& Faltinsen (2003) applied a multimodal method to simulate 
coupled ship motion and nonlinear liquid sloshing in a tank; experiments were conducted to validate the numerical program. Newman (2005) proposed that unless coupling ship motions and sloshing loads in the time domain, the liquid tank could be regarded as an extension of the exterior wet surface. Thus, no iteration between internal sloshing loads and external wave loads was needed, but it would result in larger size of matrix equation. Mitra et al. (2012) studied coupling between three dimensional sloshing and ship motion in different sea conditions, sloshing in tank was simulated based on potential flow and good agreements with experimental results were achieved. Research conducted by Zhao et al. (2015) proved potential flow with an artificial damping model can give accurate and highly efficient simulation of sloshing in the time domain.

Although multi-body floating system and liquid sloshing in vessels have been widely investigated, these two parts were mostly considered separately. The fully coupled analysis of liquid loading FLNG system in side-by-side configuration was rarely conducted. This study aims to develop an accurate and efficient numerical code to predict the dynamic responses of side-by-side arranged FLNG system. The coupling between sloshing in liquid tanks and vessels' motions are solved in the time domain, and hydrodynamic interactions between the vessels as well as motion responses under the restriction of connection system are investigated. A series of experimental tests are conducted to validate the numerical code. The research results reveal hydrodynamic interactions are sensitive to wave direction and small motions are excited when the vessel is located in the lee side. Sloshing loads will change the natural roll frequencies of vessels and lead to increased sway motions in the frequency region higher than that of natural sloshing frequency.

\section{Mathematical formulation}

In this study, an FLNG system with parallelly arranged FLNG vessel and LNG carrier is considered, and each vessel has two liquid tanks. In the numerical model, coordinate systems are defined as shown in Fig. 1 . The global coordinate system $O-X Y Z$ is earth-fixed with $Z$ axis pointing upward. Vessel-fixed coordinate systems $O_{1}-X_{1} Y_{1} Z_{1}$ and $O_{2}-X_{2} Y_{2} Z_{2}$ are respectively located in the gravity center of FLNG and LNG carrier, with $O X$ and $O Y$ pointing to the bow and portside of the vessels. The FLNG-fixed coordinate system vessel $O_{1}-X_{1} Y_{1} Z_{1}$ coincides with the earth-fixed coordinate $O-X Y Z$ initially. The four tank-fixed coordinate systems $o-x y z$ are located in the free surface center initially and parallel with the vessel-fixed coordinate system.

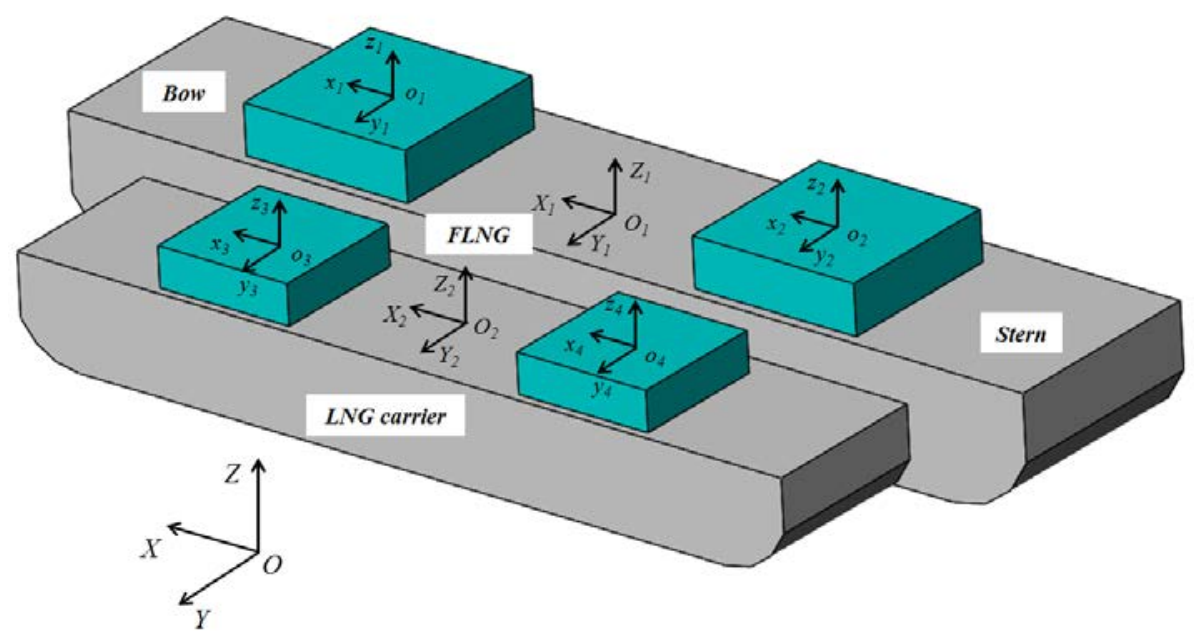

Fig. 1 Definition of coordinate systems 


\subsection{Vessels in side-by-side configuration}

Vessel motions in waves are regarded as linear system that response linearly to wave excitation. In the time domain calculation, impulsive response function method based on frequency-domain results is used in dealing with radiation forces, and solution of vessel motions in the frequency domain is required.

The linear incident wave potential can be written in a general form as:

$$
\phi_{I}(x, y, z, t)=\frac{\zeta_{a} g}{\omega} e^{k z} \sin [k(x \cos \beta+y \sin \beta)-\omega t-\sigma]
$$

where $\zeta_{a}, \omega, k, \beta, \sigma$ are the wave amplitude, frequency, wave number, heading angle and phase shift, respectively. The potential in the flow field can be regarded as the superposition of incident wave potential, radiation potentials and diffraction potential:

$$
\phi=\phi_{I}+\phi_{R i}^{F}+\phi_{R i}^{L}+\phi_{D}
$$

where $\phi_{I}$ is the incident wave potential, $\phi_{R i}^{F}$ and $\phi_{R i}^{L}$ are respectively the radiation potential corresponding to the 6 degree motions of FLNG and LNG carrier; $\phi_{D}$ is the diffraction potential. In the flow field, the potential $\phi$ satisfies the Laplace equation:

$$
\nabla^{2} \phi=0
$$

On the free surface, the linearized free surface condition is

$$
\frac{\partial \phi}{\partial z}-\frac{\omega^{2}}{g} \phi=0
$$

To account for energy dissipation in the gap between vessels and suppress unrealistic resonance in the gap, a damping-lid is applied in the gap zone (shown in Fig. 2). For free surface in the gap, a damping force proportional to particle velocity is added (Chen, 2005):

$$
f=-\frac{\varepsilon}{\omega} \nabla \phi
$$

The free surface condition in the gap zone is modified as

$$
\frac{\partial \phi}{\partial z}-\frac{\omega^{2}}{g} \phi-i \varepsilon \frac{\omega^{2}}{g} \phi=0
$$

where $\varepsilon$ is the damping-lid coefficient and normally need to be determined by experimental calibration.

On the seabed:

$$
\frac{\partial \phi}{\partial n}=0
$$

On the vessels' wet surface $S_{F}$ and $S_{L}$, the diffraction potential and incident wave potential have the relationship:

$$
\frac{\partial \phi_{D}}{\partial n}=-\frac{\partial \phi_{I}}{\partial n}
$$


The coupling radiation can be decomposed as two cases: FLNG moves with LNG carrier fixed and LNG carrier moves with FLNG fixed. Then, the boundary conditions of radiation potential on vessels' wet surfaces can be given as:

On the wet surface of FLNG $S_{F}$

$$
\frac{\partial \phi_{R i}^{F}}{\partial n}=\xi_{i}^{\&} n_{i}^{F} \quad \frac{\partial \phi_{R i}^{L}}{\partial n}=0
$$

On the wet surface of LNG carrier $S_{L}$

$$
\frac{\partial \phi_{R i}^{F}}{\partial n}=0 \quad \frac{\partial \phi_{R i}^{L}}{\partial n}=\xi_{i} n_{i}^{L}
$$

The hydrodynamic coefficients as added mass $\mu_{i j}$, radiation damping $\lambda_{i j}$ and wave forces in the frequency domain are computed using panel program HydroStar developed by Bureau Veritas (2010). Wave diffraction forces and radiation forces in the time domain can be obtained using IRF method. According to Newton's law, motion equation can be expressed as:

$$
\left[\begin{array}{cc}
M_{i j}^{F}+\mu_{i j}^{F F}(\infty) & \mu_{i j}^{F L}(\infty) \\
\mu_{i j}^{L F}(\infty) & M_{i j}^{L}+\mu_{i j}^{L L}(\infty)
\end{array}\right]\left[\begin{array}{c}
\xi_{i}(t) \\
\xi_{i}^{k}(t)
\end{array}\right]+\int_{0}^{t}\left[\begin{array}{cc}
k_{i j}^{F}(t-\tau) & k_{i j}^{F L}(t-\tau) \\
k_{i j}^{L F}(t-\tau) & k_{i j}^{L}(t-\tau)
\end{array}\right]\left[\begin{array}{c}
\xi_{i}^{L}(\tau) \\
\xi_{i}(\tau)
\end{array}\right] d_{\tau}+\left[\begin{array}{cc}
C_{i j}^{F} & C_{i j}^{F L} \\
C_{i j}^{L F} & C_{i j}^{L}
\end{array}\right]\left[\begin{array}{c}
\xi^{F}(t) \\
\xi^{L}(t)
\end{array}\right]=\left[\begin{array}{c}
\mathbf{F}_{i}^{F}(t) \\
\mathbf{F}_{i}^{L}(t)
\end{array}\right], \quad i, j=1,2, \cdots 6
$$

$$
\mathbf{F}=\mathbf{F}_{\text {wave }}(t)+\mathbf{F}_{\text {sloshing }}(t)+\mathbf{F}_{\text {connect }}(t)
$$

where $M_{i j}, \mu_{i j}(\infty), k_{i j}$ and $C_{i j}$ are the total mass of vessels (including liquid mass), infinite added mass, retardation function, and hydrostatic restoring coefficients. $\mathbf{F}_{\text {sloshing }}(t)$ and $\mathbf{F}_{\text {connect }}(t)$ are forces on vessels caused by liquid sloshing in tanks and connection system that including hawsers and fenders.

Retardation function $k_{i j}$ stands for memory effects of past motions and can be calculated based on radiation damping coefficients:

$$
k_{i j}(t)=\frac{2}{\pi} \int_{0}^{\infty} \lambda_{i j}(\omega) \cos (\omega t) d \omega
$$

Where $\lambda_{i j}$ is radiation damping in frequency domain. For the interaction between vessels, the memory effects can last for a much longer time than single vessel. 300 frequencies are adopted in the integral with truncation frequency equals 3.0rad/s. Added mass in infinite frequency and retardation function has the relationship as:

$$
\mu_{i j}(\infty)=\mu_{i j}(\omega)+\frac{1}{\omega} \int_{0}^{\infty} k_{i j}(t) \sin (\omega t) d t
$$

Viscous damping is non-negligible for vessel's roll motion, in this study, a commonly used equivalent linear damping coefficient is introduced as:

$$
b_{44}^{v}=2 \gamma \sqrt{\left(M_{44}+\mu_{44}(\infty)\right) C_{44}}
$$

Where the $\gamma$ is the ratio of viscous damping to critical damping coefficient. Soft numerical springs are used to restrict vessel's surge, sway and yaw motions so that monotonously increasing or decreasing motions can be 
prevented. The natural frequency of soft spring can be calculated as $\sqrt{C_{i i}^{\text {spring }} /\left(M_{i i}+\mu_{i i}(\infty)\right)}$, which are smaller than first mode response frequencies of vessel motions and sloshing.

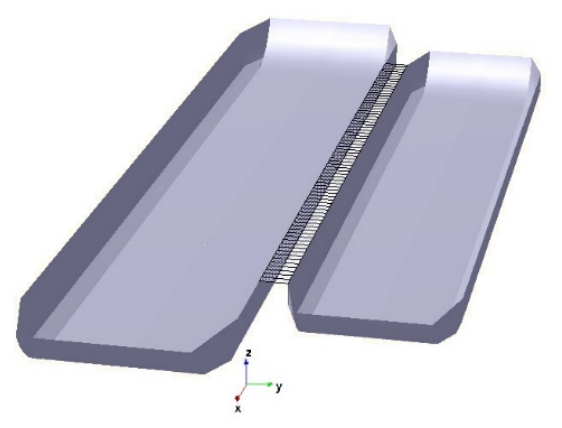

Fig. 2 Model of vessels in the calculation of frequency coefficients

\subsection{Liquid sloshing}

Internal sloshing problem in liquid tanks is solved based on potential theory by assuming that the fluid is perfect and flow irrotational. In calculation, numerical model of rectangular liquid tank with length $B$, width $W$ and water depth $D$ is built, as shown in Fig. 3, where tank 1 is taken for example.

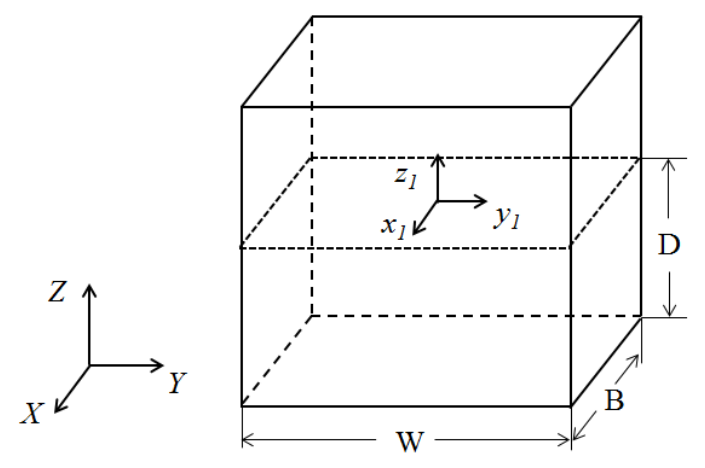

Fig. 3. Schematic of numerical tank model

For the convenience of coupling with vessel motion, sloshing problem is solved in tank-fixed coordinate. Velocity potential $\phi$ is decomposed as $\phi=\varphi+x u+y v+z w$. Boundary value problem of velocity potential $\varphi$ in tank-fixed coordinate system can be derived as:

In the fluid domain

$$
\nabla^{2} \varphi=0
$$

On wall boundaries

$$
\frac{\partial \varphi}{\partial n}=(\boldsymbol{\omega} \times \mathbf{r}) \cdot \mathbf{n}
$$

where $[u, v, w]$ and $\boldsymbol{\omega}$ are transitional velocity and rotational velocity of excitation on tank corresponding to the vessel's six degree motions. $\mathbf{n}$ is outward vector and normal to tank walls, $\mathbf{r}$ is the distance from rotation center to boundary.

On the free surface $S_{F}$, dynamic and kinematic conditions are 


$$
\begin{gathered}
\frac{\partial \varphi}{\partial t}-\boldsymbol{\omega} \times \mathbf{r} \cdot(\nabla \varphi+\mathbf{V})+x \frac{d u}{d t}+y \frac{d v}{d t}+\zeta \frac{d w}{d t}+\frac{1}{2} \nabla \varphi \cdot \nabla \varphi+k_{\theta} \mathbf{g r}_{0}=0 \\
\frac{\partial \zeta}{\partial t}+\frac{\partial \varphi}{\partial x} \cdot \frac{\partial \zeta}{\partial x}+\frac{\partial \varphi}{\partial y} \cdot \frac{\partial \zeta}{\partial y}-\frac{\partial \varphi}{\partial z}-\boldsymbol{\omega} \times \mathbf{r}_{0} \cdot \nabla \zeta=0 \\
k_{\theta}=\left[\begin{array}{ccc}
\cos \beta \cos \gamma & -\cos \beta \sin \gamma & \sin \beta \\
\sin \alpha \sin \beta \cos \gamma+\cos \alpha \sin \gamma & -\sin \alpha \sin \beta \sin \gamma+\cos \alpha \cos \gamma & -\sin \alpha \cos \beta \\
-\cos \alpha \sin \beta \cos \gamma+\sin \alpha \sin \gamma & \cos \alpha \sin \beta \sin \gamma+\sin \alpha \cos \gamma & \cos \alpha \cos \beta
\end{array}\right]
\end{gathered}
$$

where $\partial, \beta$ and $\gamma$ are the roll, pitch and yaw motions angles of the vessel, $\mathbf{g}=\left[\begin{array}{lll}0 & 0 & g\end{array}\right], \mathbf{r}_{0}=\left[\begin{array}{lll}0 & 0 & \zeta\end{array}\right], \zeta$ is the free surface elevation defined in the tank-fixed coordinate system.

Initial conditions in free surface can be expressed as $\varphi=-x u-y v-z w$ and $\eta(x, y, 0)=0$. After the solution of velocity potential, hydrodynamic pressure in wall boundaries can be achieved according to Bernoulli equation:

$$
-\frac{P}{\rho}=\frac{\partial \varphi}{\partial t}-\boldsymbol{\omega} \times \mathbf{r} \cdot(\nabla \varphi+\mathbf{V})+x \frac{d u}{d t}+y \frac{d v}{d t}+\zeta \frac{d w}{d t}+\frac{1}{2} \nabla \varphi \cdot \nabla \varphi+\mathbf{k}_{\theta} \mathbf{g r}_{0}
$$

$\varphi_{a}=\partial \varphi / \partial t$ is defined in this study and the solution of $\varphi_{a}$ can be regarded as a similar boundary value problem as the solution of $\varphi$. In tank-fixed coordinate system, the governing equations of $\varphi_{a}$ can be obtained as:

In the fluid domain

$$
\nabla^{2} \varphi_{a}=0
$$

On the free surface

$$
\varphi_{a}=-x \frac{d u}{d t}-y \frac{d v}{d t}-\zeta \frac{d w}{d t}-\frac{1}{2} \nabla \varphi \cdot \nabla \varphi-\mathbf{k}_{\theta} \mathbf{g r}_{0}+(\boldsymbol{\omega} \times \mathbf{r}) \cdot(\nabla \varphi+\mathbf{V})
$$

On wall boundaries

$$
\frac{\partial \varphi_{t}}{\partial n}=\mathbf{n} \cdot\left[\& \ll \mathbf{r}+2 \boldsymbol{\omega} \times \mathbf{V}_{r}+\boldsymbol{\omega} \times(\boldsymbol{\omega} \times \mathbf{r})-(\nabla \varphi \cdot \nabla) \nabla \varphi\right]+\mathbf{n} \cdot \nabla[(\boldsymbol{\omega} \times \mathbf{r}) \cdot(\nabla \varphi+\mathbf{V})]
$$

where $\mathbf{V}_{r}$ is the velocity of fluid observed in tank-fixed coordinate system.

After solving $\varphi_{a}$, sloshing loads on vessels can be obtained. The time-derivative of potential on the free surface is obtained as:

$$
\frac{d \varphi}{d t}=\frac{\partial \zeta}{\partial t} \frac{\partial \varphi}{\partial y}-x \frac{d u}{d t}-y \frac{d \nu}{d t}-\zeta \frac{d w}{d t}-\frac{1}{2} \nabla \varphi \cdot \nabla \varphi-\mathbf{k}_{\theta} \mathbf{g r}_{0}+(\boldsymbol{\omega} \times \mathbf{r}) \cdot(\nabla \varphi+\mathbf{V})
$$

For the convenience of mesh updating during calculation, free surface is updated vertically in the tank-fixed coordinate:

$$
\frac{d \zeta}{d t}=\frac{\partial \varphi}{\partial y}-\frac{\partial \zeta}{\partial x} \frac{\partial \varphi}{\partial x}-\frac{\partial \zeta}{\partial y} \frac{\partial \varphi}{\partial y}+(\boldsymbol{\omega} \times \mathbf{r}) \cdot \nabla \zeta
$$


Artificial damping model is used to account for energy dissipation caused by fluid viscosity. A damping force proportional to particle velocity with an opposite direction is assumed on the free surface. With damping coefficient $\mu$, Euler's equation can be expressed as:

$$
\frac{D w}{D t}=-\frac{1}{\rho} \nabla p-g \nabla \zeta-\mu \nabla \varphi
$$

The sloshing model is solved numerically in BEM, details about numerical simulation can be found in Zhao et al. (2017 b).

\subsection{Connection system}

The relative motions between vessels are restricted by connection system to guarante the safety of offloading arm and avoid collision between vessels during the offloading operation. Loads on connection system $\mathbf{F}_{\text {connect }}$ including $\mathbf{F}_{\text {hawser }}$ and $\mathbf{F}_{\text {fender }}$ are related to the relative motions between vessels. Hawsers and fenders are numerically simulated as linear stiffness systems. A sketch of hawsrs and fenders are presented in Fig. 4 to illustrate the solution of $\mathbf{F}_{\text {connect }}$.

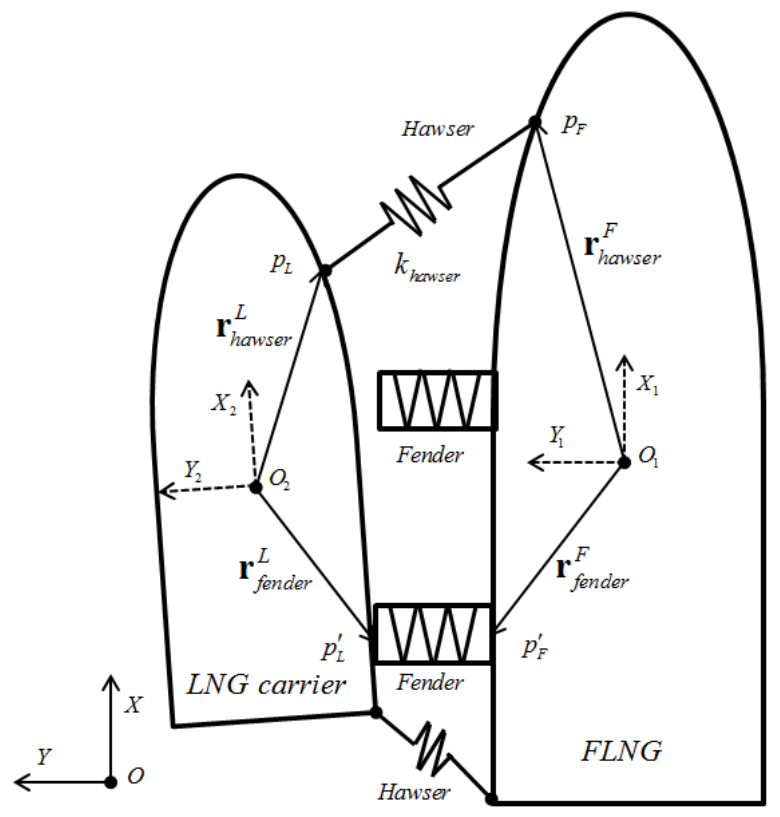

Fig. 4. Schematic of connection system

The hawser can only be stretched and tension on hawser can be given as:

$$
\begin{array}{ll}
\mathbf{F}_{\text {hawser }}=k_{\text {hawser }} \cdot \delta l_{\text {hawser }} \cdot \mathbf{n}_{\text {hawser }} & \delta l_{\text {hawser }}>0 \\
\mathbf{F}_{\text {hawser }}=0 & \delta l_{\text {hawser }}<0
\end{array}
$$

where $k_{\text {hawser }}$ and $\delta l_{\text {hawser }}$ are the stiffness coefficient and elongation of hawser, $\mathbf{n}_{\text {hawser }}$ stands for the hawser direction that from FLNG to LNG carrier. For each hawser, $p_{F}(x, y, z)$ and $p_{L}(x, y, z)$ are points where the hawser is attached to FLNG vessel and LNG carrier. The distance between $p_{F}$ and $p_{L}$ is the cooresponding hawser length $l_{\text {hawser }}$. The motion of $p_{F}$ and $p_{L}$ in global coordinate system $O-X Y Z$ can be described as

$$
p_{F}=\xi_{i}^{F}+k_{\theta}^{F} \mathbf{r}_{\text {hawser }}^{F} \quad i=1,2,3
$$




$$
p_{L}=\xi_{i}^{L}+k_{\theta}^{L} \mathbf{r}_{\text {hawser }}^{L} \quad i=1,2,3
$$

where $k_{\theta}^{L}$ and $k_{\theta}^{L}$ are related to vessels' rotational motions, and have the same form as $k_{\theta}$ in equation (20). The superscript ' $F$ ' and ' $L$ ' stand for FLNG and LNG carrier, respectively. The elongation and normal direction of hawser can be obtained as:

$$
\begin{aligned}
& \delta l_{\text {hawser }}=\left\|\operatorname{umu}_{p_{F} p_{L}}\right\|-l_{\text {initial }} \\
& \mathbf{n}=\frac{\underset{p_{E} p_{f}}{\operatorname{coum}}}{\left\|p_{F} p_{L}\right\|}
\end{aligned}
$$

where $l_{\text {initial }}$ is the length of hawser at the initial condition in still water and can be solved as:

$$
l_{\text {initial }}=\left\|\mathbf{r}_{\text {hawser }}^{L}+\mathbf{r}_{\text {rel }}-\mathbf{r}_{\text {hawser }}^{F}\right\|-l_{\text {pretension }}
$$

where $\mathbf{r}_{\text {hawser }}^{F}$ and $\mathbf{r}_{\text {hawser }}^{L}$ are the coordinates of $p_{F}$ and $p_{L}$ that described in vessel-fixed coordinate systems $O_{1}-X_{1} Y_{1} Z_{1}$ and $O_{2}-X_{2} Y_{2} Z_{2}, \quad \mathbf{r}_{r e l}=O_{1} O_{2}$ is the distance between origin of $O_{1}-X_{1} Y_{1} Z_{1}$ and $O_{2}-X_{2} Y_{2} Z_{2}$ that described in global coordinate system $O-X Y Z$. $l_{\text {pretension }}$ is the elongation of hawser that caused by the pretension of hawser, and can be given as

$$
l_{\text {pretension }}=\frac{F_{\text {pretension }}}{k_{\text {hawser }}}
$$

Then, forces applied on FLNG and LNG carrier by hawser can be given as

$$
\mathbf{F}_{\text {hawser }}^{F}=-\mathbf{F}_{\text {hawser }}^{L}=\mathbf{F}_{\text {hawser }}
$$

The hawser forces need to be transferred to vessel-fixed coordinate system before the calculaiton of the moment applied on vessels. Thus, the moment exerted on FLNG and LNG carrier can be given as

$$
\begin{aligned}
& \mathbf{M}_{\text {hawser }}^{F}=\mathbf{r}_{\text {hawser }}^{F} \times\left(k_{\theta}^{F}\right)^{-1} \mathbf{F}_{\text {hawser }}^{F} \\
& \mathbf{M}_{\text {hawser }}^{L}=\mathbf{r}_{\text {hawser }}^{L} \times\left(k_{\theta}^{L}\right)^{-1} \mathbf{F}_{\text {hawser }}^{L}
\end{aligned}
$$

where $k_{\theta}^{F}$ and $k_{\theta}^{L}$ are in the form of (20), and correspond to the rotation motion of FLNG and LNG carrier, respectively.

The fender can only be compressed and reaction force can be given as

$$
\mathbf{F}_{\text {fender }}=k_{\text {fender }} \cdot \delta l_{\text {fender }} \cdot \mathbf{n} \quad \delta l_{\text {fender }} \leq 0
$$

where $\delta l_{\text {fender }}$ is the deformation of fender under compression.

Unlike hawsers that attachted to the two vessels, the fenders are floating in the free surface between vessels. In this study, fender is simplified to be fixed to FLNG vessel on the free surface position $p_{F}^{\prime}$ and perpendicular to the FLNG hull surface. Thus, position $p_{L}^{\prime}$ where fender reaction force exerts on LNG vessel varies when relative 
motions between vessels occur. Assumeing normal direction of fender in initial condition $\mathbf{n}_{\text {fender }}$, the fender length $\kappa$ and coordinates of $p_{L}^{\prime}$ stasify the following relationship:

$$
\xi_{i}^{F}+k_{\theta}^{F} \mathbf{r}_{\text {fender }}^{F}+\kappa k_{\theta}^{F} \mathbf{n}_{\text {fender }}=\xi_{i}^{L}+k_{\theta}^{L} \mathbf{r}_{\text {fender }}^{L}+\mathbf{r}_{\text {rel }} \quad i=1,2,3
$$

on which, $p_{L}^{\prime}(y)$ is known for $p_{L}^{\prime}$ located in the starboard of LNG carrier. $p_{L}^{\prime}(x), p_{L}^{\prime}(z)$ and fender length $\kappa$ can be solved according to this linear equaiton set.

After the solution of fender length, fender force can be obtaned according to (38). Moment exerted on FLNG and LNG carrier by fender can be given as:

$$
\begin{aligned}
& \mathbf{M}_{\text {fender }}^{F}=\mathbf{r}_{\text {fender }}^{F} \times\left(k_{\theta}^{F}\right)^{-1} \mathbf{F}_{\text {fender }}^{F} \\
& \mathbf{M}_{\text {fender }}^{L}=\mathbf{r}_{\text {fender }}^{L} \times\left(k_{\theta}^{L}\right)^{-1} \mathbf{F}_{\text {fender }}^{L}
\end{aligned}
$$

\subsection{Coupling solution}

The complex coupling effects in FLNG system make the direct solution of (11) in the time domain rather complicated. In the present study, an iteration strategy is adopted to couple the responses of liquid sloshing and connection system with vessels' motions.

In the iteration process, as shown in Fig. 5, vessel motions serve as the excitation on the liquid tank, and the relative motions between vessels determine the loads on connection system. The sloshing and connection system loads work as the excitation forces on vessels' motions. The wave action part is solved using IRF method that described in Section 2.1 and is not affected by the time domain calculation. The solution of liquid sloshing and connection system parts are described in Section 2.2 and 2.3, respectively. In the time domain calculation, iteration calculation between vessels' motions and loads from sloshing and connection system is conducted in each step until convergence is obtained, which means the balance between forces and motions in (11) is achieved. After the iteration calculation, both vessel motions and internal sloshing are updated, then calculation proceeds the next time step.

It should be noted $F_{\text {sloshing }}(t)$ is solved in tank-fixed coordinate system and needs to be transferred to the coordinate system of vessel motions. In addition, inertia forces of liquid have been considered in the first term in left side of equation (11) and need to be excluded from $F_{\text {sloshing }}(t)$ in the coupling calculation. 
Iteration calculation

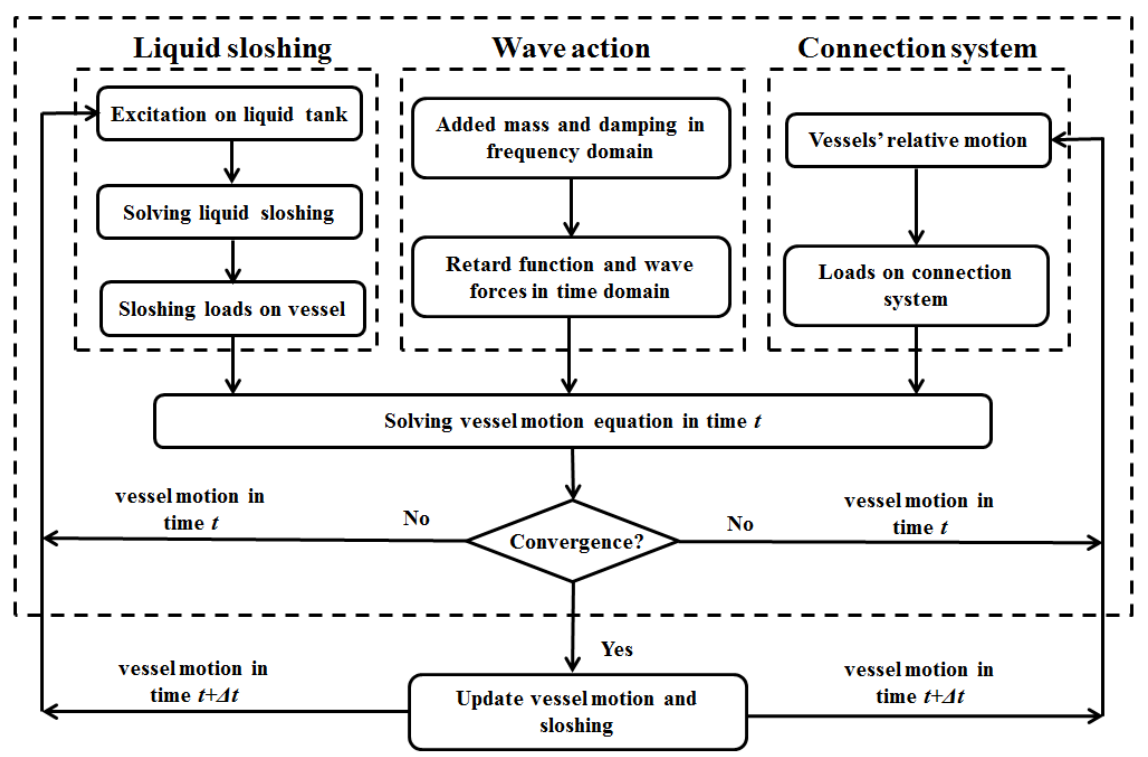

Fig. 5 Flow chart of the numerical code in the simulation of FLNG system

\section{Model tests}

A model test was conducted to validate the proposed numerical code. The model test was conducted in Deepwater Offshore Basin at Shanghai Jiao Tong University at a model scale of 1:60. The basin is $50 \mathrm{~m}$ long, $40 \mathrm{~m}$ wide and $10 \mathrm{~m}$ in depth. Wave makers are installed in the two adjacent sides of the basin and oblique waves can be produced. The other two adjacent sides are installed with wave absorber equipment to prevent the wave reflection.

\subsection{Vessels model}

Arrangement of FLNG system in side-by-side configuration is presented in Fig. 6. The FLNG and LNG carrier are placed in parallel with initial gap $6 \mathrm{~m}$. The two vessels have similar profiles but different size. For each vessel, two plexiglass rectangular tanks are installed symmetrically about the mid-ship section. The main particulars of vessels and tanks are listed in Table 1, FLNG and LNG carrier are respectively $66.7 \%$ and $60 \%$ filled. Solid loading tests are also included in the experiment for comparison. The solid and liquid loading conditions have same inertia particulars when assume the liquid in tanks is frozen. Wave probes are installed in the liquid tanks close to the bow (T1 to T10 in Fig. 6 (a)) to capture sloshing wave elevation in representative positions. Wave probes T11 to T13 are fixed to vessels to measure wave elevation around vessels.

In the model testes, vessels are restricted with soft horizontal mooring lines (shown in Fig. 7) to prevent motions caused by drift forces. The soft stiffness of the horizontal mooring lines make sure the natural frequency of mooring system is very low and has slight effects on vessels' first order motions. The horizontal mooring forces are not considered in the numerical model described in section 2 for only first order wave forces are adopted. 


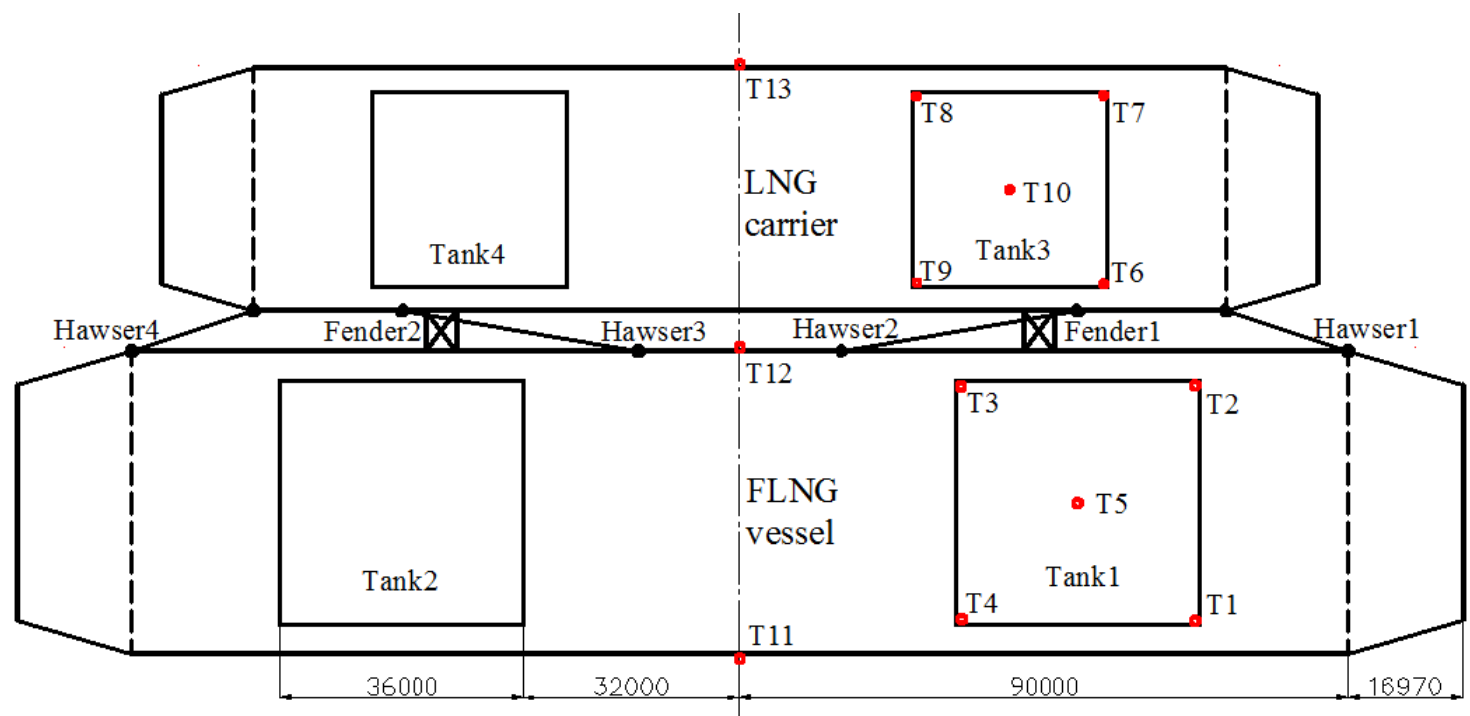

(a) Top view

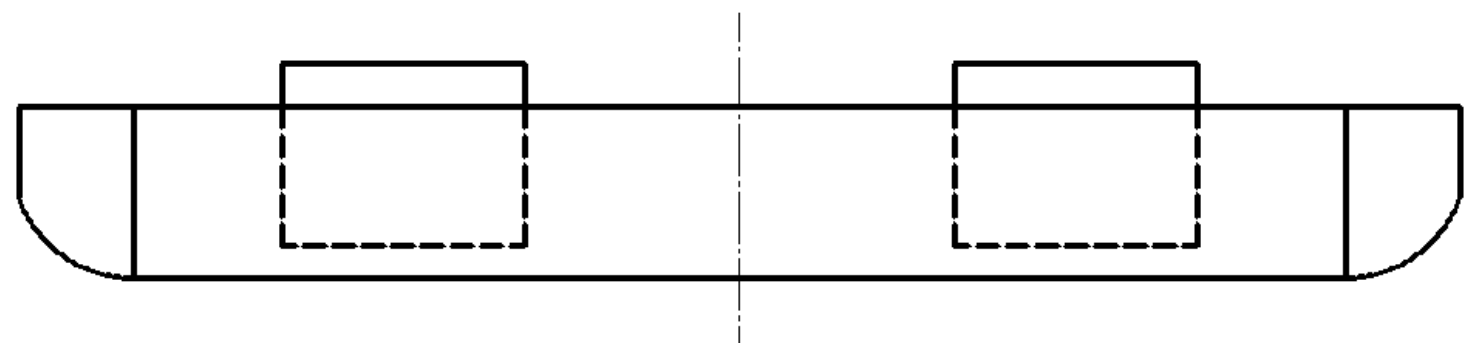

(b) Side view of FLNG

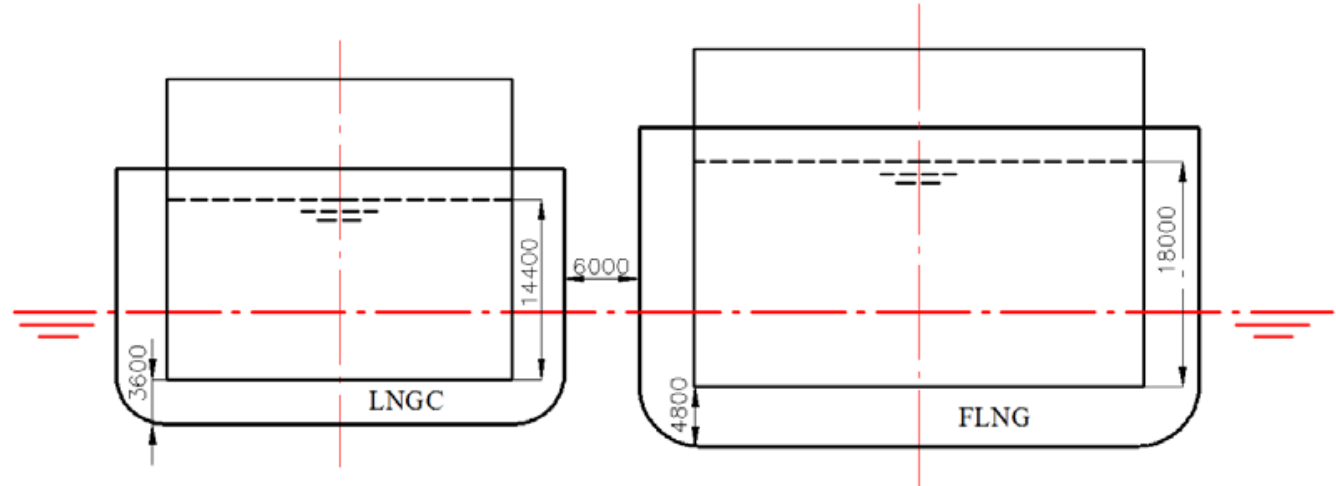

(c) Front view

Fig. 6 Profiles of vessels in side-by-side arranged FLNG system (unit: mm) 


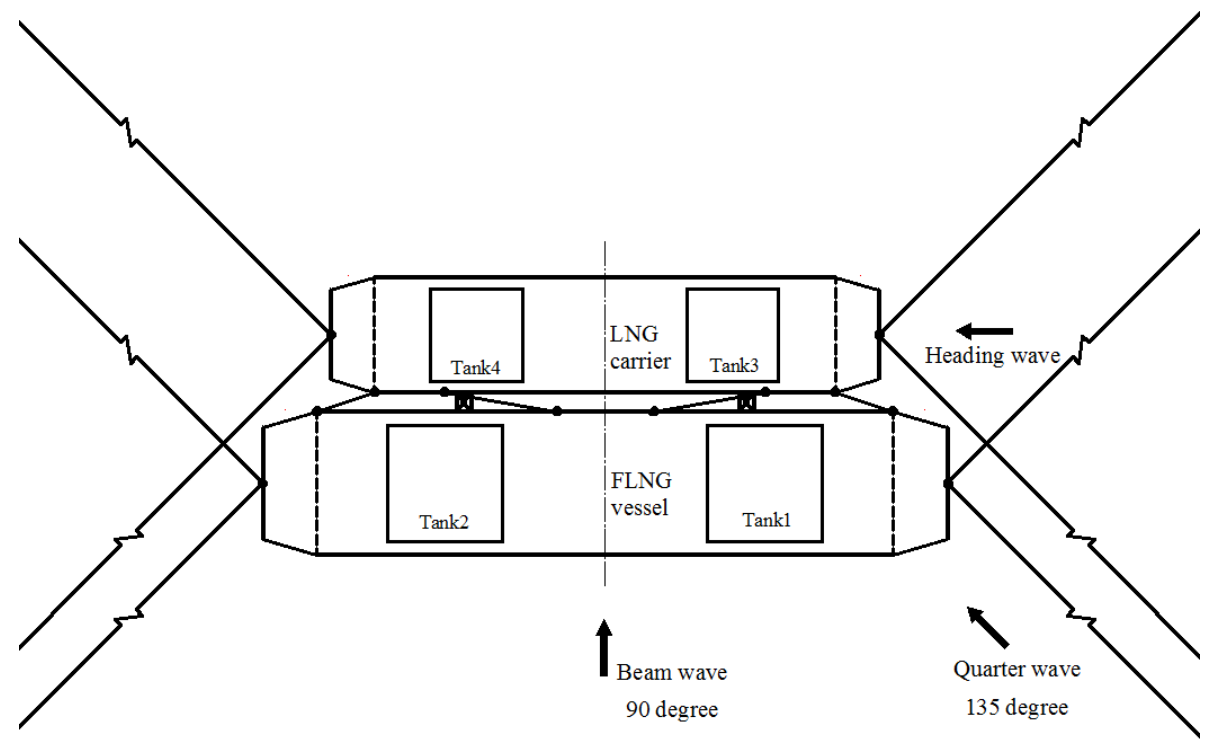

Fig. 7 Mooring configuration side-by-side arranged vessels

Table 1 Main particulars of vessels and LNG tanks

\begin{tabular}{ccc}
\hline & Main particulars & \\
\hline & FLNG & LNG carrier \\
Length (m) & 213.9 & 171.2 \\
Width (m) & 44.8 & 35.8 \\
Depth (m) & 25.5 & 20.4 \\
Draft (m) & 10.8 & 9 \\
Displacement (t) & 98923.1 & 52821.3 \\
Roll gravity radius (m) & 16 & 10.2 \\
Pitch gravity radius (m) & 60 & 50 \\
Yaw gravity radius (m) & 60 & 50 \\
Vertical gravity center (m) & 13.8 & 12 \\
Tank length (m) & 36 & 29.44 \\
Tank width (m) & 36 & 29.44 \\
Tank height (m) & 27 & 24 \\
\hline
\end{tabular}

\subsection{Connection system}

A simplified connection system that consists of four hawsers and two fenders are adopted in this study. Both the hawsers and the fender are simulated as linear stiffness system. Though the nonlinearity of fender will become significant under large deformation, when the compression on fender is limited, the linearized fender mode has been proved to be feasible (Zhao et, al. 2017 a) for the less violent motions of FLNG system. The connection system is attached to the port of FLNG and starboard of LNG carrier, respectively. Hawsers are located in the upper deck and fenders are located in the water line position and fixed to FLNG vessel. Coordinates and stiffness of hawsers and fenders are listed in Table 2. In the initial condition, the hawsers are stretched with a pretension of 350 $\mathrm{kN}$, this pretension is balanced by the reaction forces of fenders and static restore forces of vessels.

Table 2 Main particulars of FLNG vessel and LNG tank

\begin{tabular}{|c|c|c|}
\hline $\begin{array}{l}\text { Location in FLNG } \\
\text { (FLNG-fixed coordinate system) }\end{array}$ & $\begin{array}{c}\text { Location in LNG carrier } \\
\text { (LNG carrier-fixed coordinate } \\
\text { system) }\end{array}$ & Stiffness \\
\hline$y(\mathrm{~m})$ & $y(\mathrm{~m})$ & $K(\mathrm{kN} / \mathrm{m})$ \\
\hline
\end{tabular}




\begin{tabular}{lccccccc} 
Hawser 1 & 90.0 & 22.4 & 14.7 & -74.0 & -17.9 & 11.4 & 4689.0 \\
Hawser 2 & 15.0 & 22.4 & 14.7 & -50.0 & -17.9 & 11.4 & 2690.0 \\
Hawser 3 & -15.0 & 22.4 & 14.7 & 50.0 & -17.9 & 11.4 & 2690.0 \\
Hawser 4 & -90.0 & 22.4 & 14.7 & 74.0 & -17.9 & 11.4 & 4689.0 \\
Fender 1 & 46.0 & 22.4 & -3.0 & 46.0 & -17.9 & -1.8 & 885.6 \\
Fender 2 & -46.0 & 22.4 & -3.0 & -46.0 & -17.9 & -1.8 & 885.6 \\
\hline
\end{tabular}

\subsection{Sea states}

White noise waves are adopted in the model tests to obtain FLNG system responses in a wide frequency range. The white noise waves used have significant wave amplitude of $1.5 \mathrm{~m}$ to meet the linear wave assumption. Wave period ranges from $5 \mathrm{~s}$ to $30 \mathrm{~s}$ to cover the main response periods of vessels' motion and liquid sloshing in tanks. Different wave directions (defined in Fig. 7) including beam waves (90 and 270 degree), oblique waves (135 and 225 degree) and heading wave (180 degree) are considered in the model tests. White noise waves are calibrated before the model test by measuring wave elevation histories in the center of basin using wave probe. After FFT calculation, the measured wave spectra are obtained and comparison with target wave spectra is presented in Fig. 8.

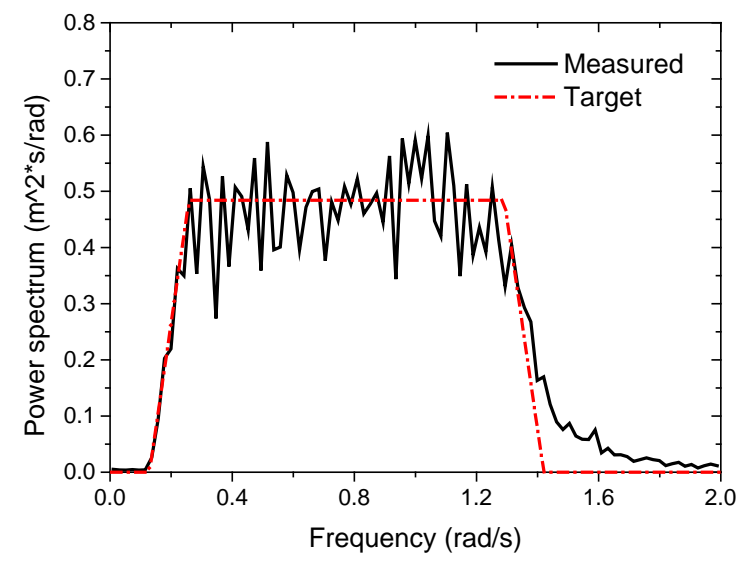

Fig. 8 Spectra of white noise wave in beam sea condition

\section{Comparison and validation}

The developed numerical code is validated in this section. The numerical code in predicting liquid sloshing under forced excitation, coupling between sloshing and ship motions, interactions between two vessels are separately validated by previous studies and experimental results. All the numerical simulations are conducted in the time domain; some results are normalized by excitation amplitude or wave amplitude and presented in the form of response amplitude operators (RAO).

\subsection{Liquid sloshing}

In this subsection, numerical predictions of sloshing under forced excitations are validated. Natural sloshing frequencies of three dimensional rectangular tank described in Fig. 3 can be expressed analytically as:

$$
\omega_{m n}=\sqrt{\pi \sqrt{\left(\frac{m^{2}}{B^{2}}+\frac{n^{2}}{W^{2}}\right)} \tanh \left(\pi \sqrt{\left(\frac{m^{2}}{B^{2}}+\frac{n^{2}}{W^{2}}\right)}\right)}
$$

where $m, n$ are positive integers. For sloshing cases with different tanks size and excitation, convergences analyses are conducted to guarantee the mesh size and time step convergence. Firstly, sloshing responses in three dimensional liquid tank are compared with calculation results of Wu et, al. (1998) in FEM method. Parameters of 
tank are $B=W=4$, displacements of tank in $x$ and $y$ directions are in sinusoidal form as $d_{x}(t)=A_{x} \sin \left(\omega_{x} t\right)$ and $d_{y}(t)=A_{y} \sin \left(\omega_{y} t\right)$ with $A_{x}=0.0372, \omega_{x}=\omega_{10}, A_{y}=0.0182, \omega_{y}=\omega_{01}$. Mesh numbers along tank's width, length and water depth are set as $14 \times 14 \times 8$, and time step is set as 0.01 s. Wave elevation histories in two corners are presented in Fig. 9 and good agreements are obtained.

Secondly, free surface elevation in sloshing under rotationally forced excitations is validated. A two dimensional tank subjected to rotational excitation solved by Nakayama \& Washizu (1981) is chosen. The tank has parameters as $B=0.9 \mathrm{~m}, D=0.6 \mathrm{~m}$. Rotational excitation on the tank is applied in cosine form as $A_{\theta x}(t)=\theta_{x} \cos \left(\omega_{\theta x} t\right)$, where $\theta_{x}=0.8 \mathrm{deg}$ and $\omega_{\theta x}=5.5 \mathrm{rad} / \mathrm{s}$. In the proposed numerical program, a numerical tank with $B \times W \times D=0.9 m \times 0.3 m \times 0.6 m$ is built and mesh numbers are set as $18 \times 6 \times 12$. Wave elevation histories in the corner $(B / 2,-D / 2)$ are presented in Fig. 10 and good agreement is obtained. Moreover, sloshing loads on tank wall due to rotational excitation are also validated. Experimental and numerical studies conducted by Chen et, al. (2013) are chosen. Dimensions of the liquid tank are $B \times W \times D=1 \mathrm{~m} \times 1 \mathrm{~m} \times 0.3 \mathrm{~m}$ and mesh numbers are set as $15 \times 15 \times 5$ in the present simulation. Rotational excitation on the tank is in sinusoidal form as $A_{\theta x}(t)=\theta_{x} \sin \left(\omega_{\theta x} t\right)$ with $\theta_{x}=5 \mathrm{deg}$. Two cases with $\omega_{\theta x}=0.95 \mathrm{rad} / \mathrm{s}$ and $\omega_{\theta x}=3.09 \mathrm{rad} / \mathrm{s}$ are selected. Pressure load histories on the left wall that $0.1 \mathrm{~m}$ form initial free surface are captured. Fig. 11 shows that present numerical results in $\omega_{\theta x}=0.95 \mathrm{rad} / \mathrm{s}$ agree well with experimental and numerical results obtained by Chen et, al. (2013). When excitation increases to $\omega_{\theta x}=3.09 \mathrm{rad} / \mathrm{s}$, larger sloshing is induced and pressure discrepancy appears for the free surface of liquid are not so regular as in mild sloshing case. The general tendency and amplitude of sloshing pressure are well simulated, which proves the validation of the proposed numerical program.

It should be noted that the applicability of proposed numerical program is limited to sloshing with single valued free surface profile. Violent sloshing that includes wave breaking and overturning are not considered in the present study. Thus, extreme shallow loading conditions that tank bottom may be out of water are also beyond the capability of the present model.
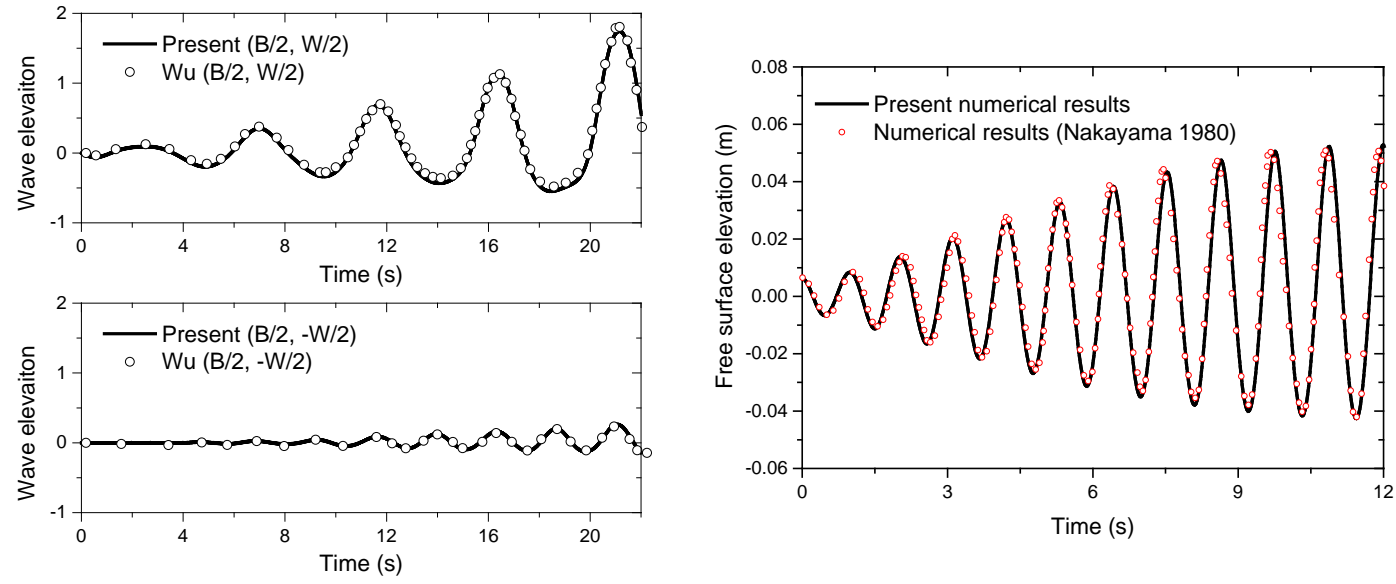

Fig. 9. 3D sloshing wave elevation histories

Fig. 10. Wave elevation histories due to rotational excitation 

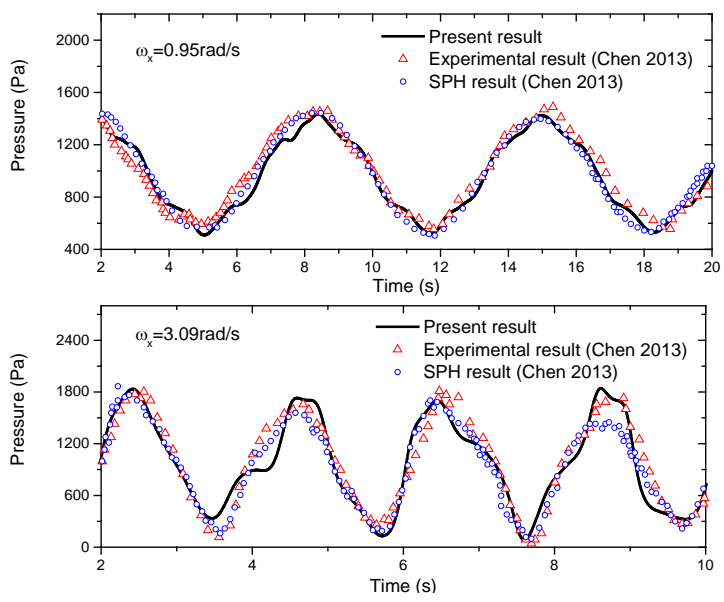

Fig. 11. Pressure histories due to rotational excitation

\subsection{Coupling of liquid loading vessel}

Validaiton of coupling calculaiton of liquid loading single vessel is conducted using experimental resutls. Test results of solid and liquid loading conditions serve as the validation of vessel motion calculation and coupling calculation, respectively.

Firstly, experimental tests conducted by Rognebakke and Faltinsen (2003) are selected for comparison. In Rognebakke's experiment, a rectangular hull contains two liquid tanks is restricted to sway motion in waves. Responses of solid loading condition and liquid loading condition with two tanks $0.186 \mathrm{~m}$ filled are selected in the comparison with numerical results. Mesh numbers along the length, width and water depth of each liquid tank are set as $8 \times 18 \times 10$. Fig. 12 shows the comparison of sway RAOs, and good agreements are obtained. Then, experimental results of the solid and liquid loading FLNG vessel under beam sea condition are used. Comparison of vessel's motions and sloshing wave elevation in tank are presented in Fig. 13. $\mu=0.1$ is selected to account for energy dissipation in sloshing, and good agreements in vessel's motions and sloshing responses are obtained. The good agreements between numerical and experimental results prove that the proposed numerical code can give good prediction of the coupling in liquid loading vessels. It can be pointed out that sway motions of liquid loading decrease to almost 0 in the natural sloshing frequencies. The two sloshing peaks appear near natural roll frequency and natural sloshing frequency, it can be deduced the sway and roll motions are respectively the main excitation of sloshing peaks.

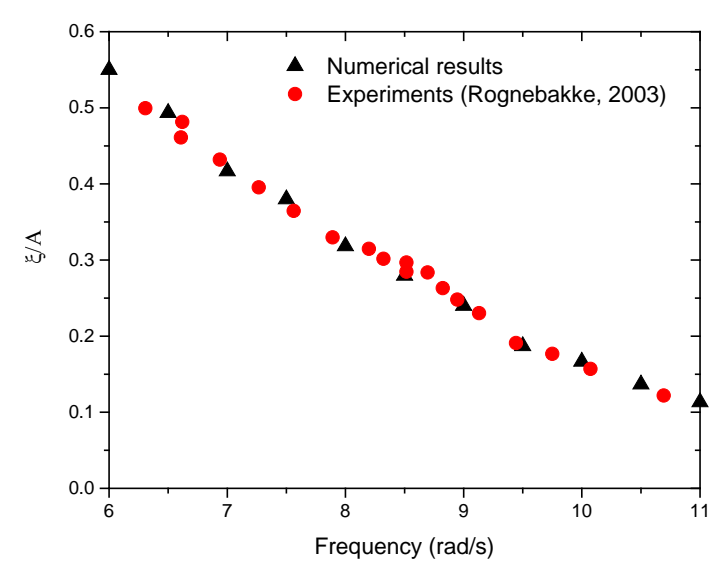

(a) Solid loading

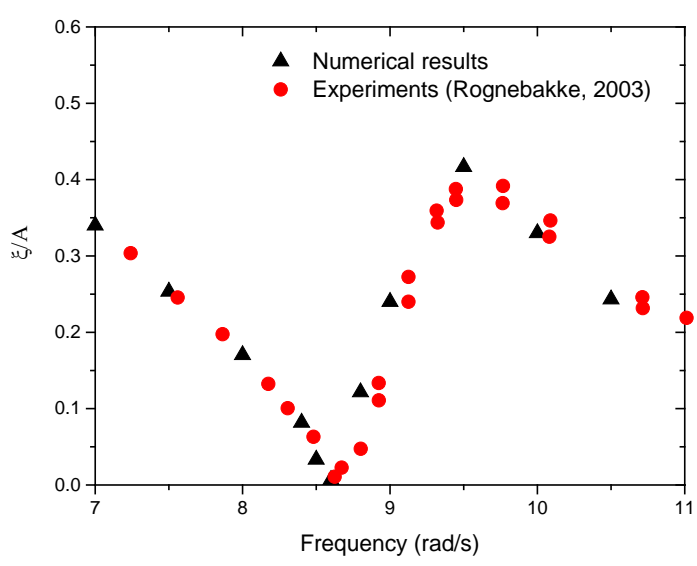

(b) liquid loading

Fig. 12. RAOs of sway motion in solid and liquid loading conditions 

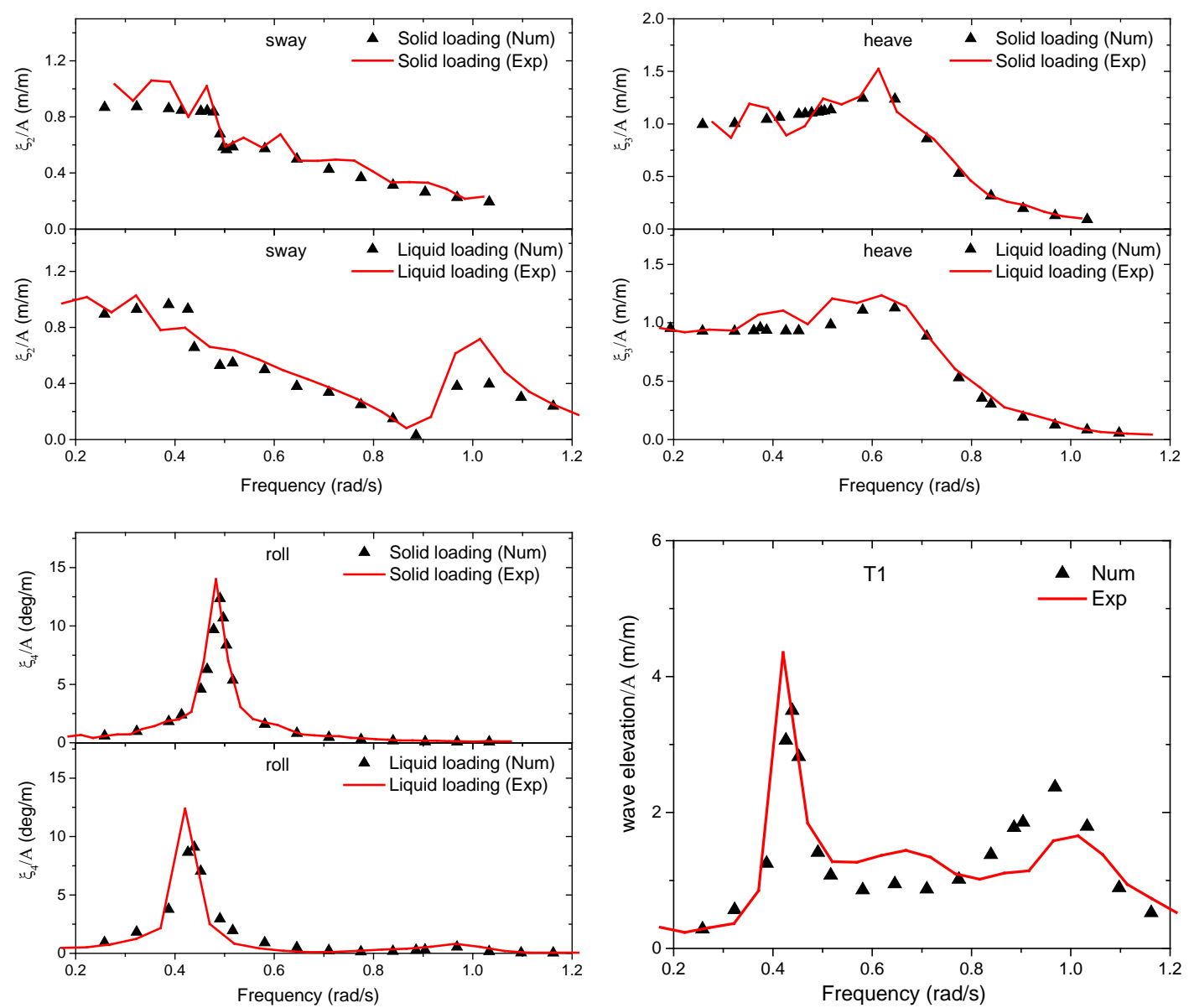

Fig. 13 RAOs of vessel motions and sloshing elevation in T1 under beam sea

\subsection{Resonant responses in the gap zone}

Damping coefficient $\varepsilon$ on the free surface of the gap zone is crucial to hydrodynamic coefficients of side-by-side arranged FLNG system. In this study, wave elevation in the gap that sensitive to damping value is selected to validate the value of $\varepsilon$.

Experimental results of wave elevation in T12 (shown in Fig. 6(a)) are used to validate selection of $\varepsilon$. As wave probe T12 is fixed to the larboard of FLNG vessel, wave elevation caused by vessel's motions need to be excluded. Different values of $\varepsilon$ are applied in the calculations in frequency domain, comparisons of wave elevation in the middle of the gap with experimental results are presented in Fig. 14. Three wave directions are selected to check the sensitivity of damping value to wave directions. Response peaks that correspond to the natural frequencies of gap resonances can be observed, and the damping lid model can suppress the resonant wave elevation in the gap successfully. The damping lid has slight effects on responses in the low frequency range that away the resonant frequency. Though the RAOs of wave elevation differ obviously under different wave directions, good agreements between numerical and experimental results can be obtained when $\varepsilon=0.05$. In the calculation of hydrodynamic coefficients of vessels in frequency domain, $\varepsilon=0.05$ is selected. 

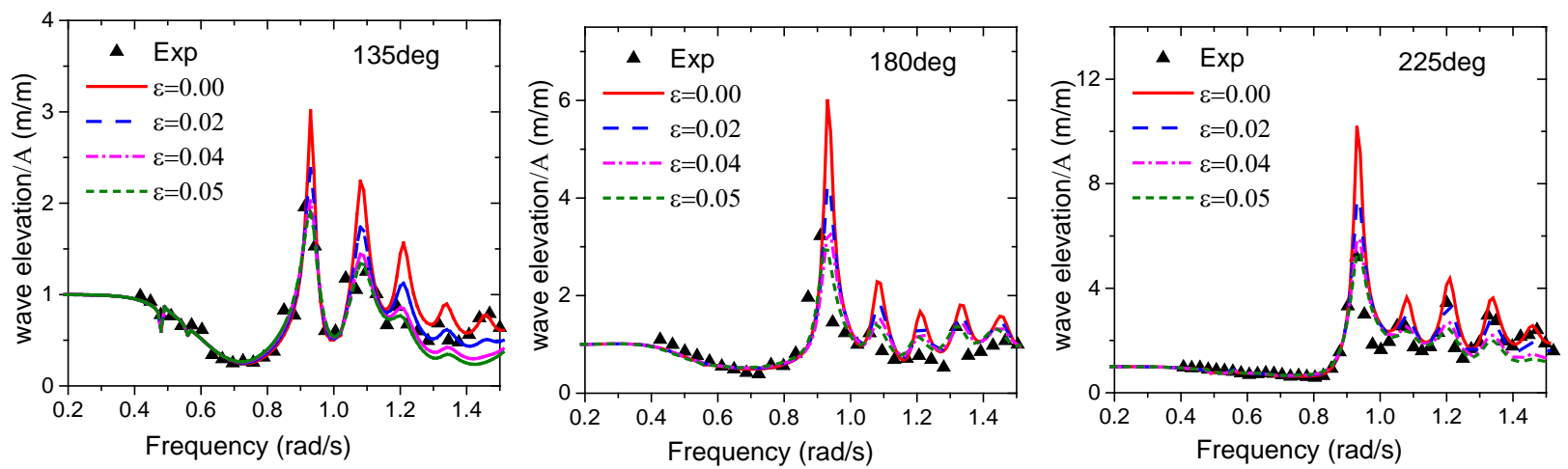

Fig. 14 Comparison of wave elevation in the gap center under different wave directions

The resonant frequencies in the gap obtained from the numerical and experimental results have good agreements. Molin $(2001,2002)$ gave the general solution of resonant frequencies in open-ended moonpool. For moonpool has the size of length $L$, width $B$ and draft $D$, resonant frequencies can be calculated as:

$$
\omega_{m n}=\sqrt{g v_{m n} \frac{1+J_{m n} \tanh \left(v_{m n} H\right)}{J_{m n}+\tanh \left(v_{m n} H\right)}}
$$

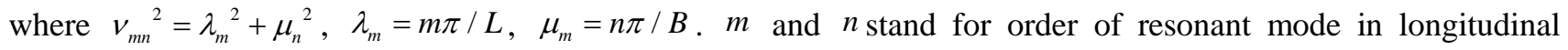
and lateral direction, respectively. In the narrow gap with $B / L$ is rather small, response along the width of the gap is in the form of piston type. With the increase of $B$ response translate into types with different modes. For FLNG system, the gap is rather small compared with length of vessels. Resonant frequencies in that case correspond to $n=0$ in (43) and $J_{m 0}$ can be written as (Molin et al., 2002):

$$
J_{m 0}=\frac{2}{m \pi^{2} r}\left\{\int_{0}^{1} \frac{r^{2}}{u^{2} \sqrt{u^{2}+r^{2}}}\left[1+2 u+(\mathrm{u}-1) \cos (m \pi u)-\frac{3}{m \pi} \sin (m \pi u)\right] d u-\frac{1}{\sin \theta}+1+2 r \ln \frac{1+\cos \theta}{1-\cos \theta}\right\}
$$

where $r=B / L, \tan \theta=1 / r$.

In Table 3, gap resonant frequencies under four wave directions are presented. Analytical results are also obtained for comparison, in which $B=6 \mathrm{~m}$ and $L=160 \mathrm{~m}$ is set as the length of LNG carrier. Considering Neumann condition defined in the bottom of gap Molin (2001), it is more reasonable to define $D=9 \mathrm{~m}$ according to the draft of LNG carrier. Table 3 shows the shift of resonant frequencies exists between the analytical and experimental results. Gap resonance between floating bodies tends to have larger frequencies for the radiation effects of vessels, and this tendency can also been found in model tests conducted by Sun et al. (2010). Besides, the difference size between the two vessels will cause complex diffraction effects, which also contribute to the differences between the discrepancies in Table. 3 .

Table 3 Resonant frequencies in gap under different wave directions

\begin{tabular}{cccc}
\hline Mode & $\omega_{10}$ & $\omega_{30}$ & $\omega_{50}$ \\
\hline Ana (D=9m) & 0.840 & 0.979 & 1.122 \\
\hline Exp(135deg) & 0.910 & 1.072 & 1.191 \\
Exp(180deg) & 0.896 & 1.064 & 1.198 \\
$\operatorname{Exp}(225 \mathrm{deg})$ & 0.905 & 1.033 & 1.189 \\
\hline
\end{tabular}




\subsection{Vessels' motions in side-by-side configuration}

Using hydrodynamic coefficients with considering damping lid in the gap zone, vessels' motions under different wave directions are validated. Experimental results of solid loading FLNG system under oblique (135 degree) and head waves are selected to validate the numerical code. RAOs of vessels' motions are presented in Fig. 15 and Fig. 16, good agreements between experimental and numerical results are obtained, which indicates the frequency coefficients calculated can guarantee good results in time domain. The frequency region smaller than $0.2 \mathrm{rad} / \mathrm{s}$ is not considered as horizontal mooring system plays dominant role in this region, especially for vessels' sway, swan and yaw motions. The vessels' motions are considered in linear range, and the viscous model in roll mode can give good prediction of the vessels' roll motion responses in the natural roll frequencies for the nonlinear viscous is not obvious.
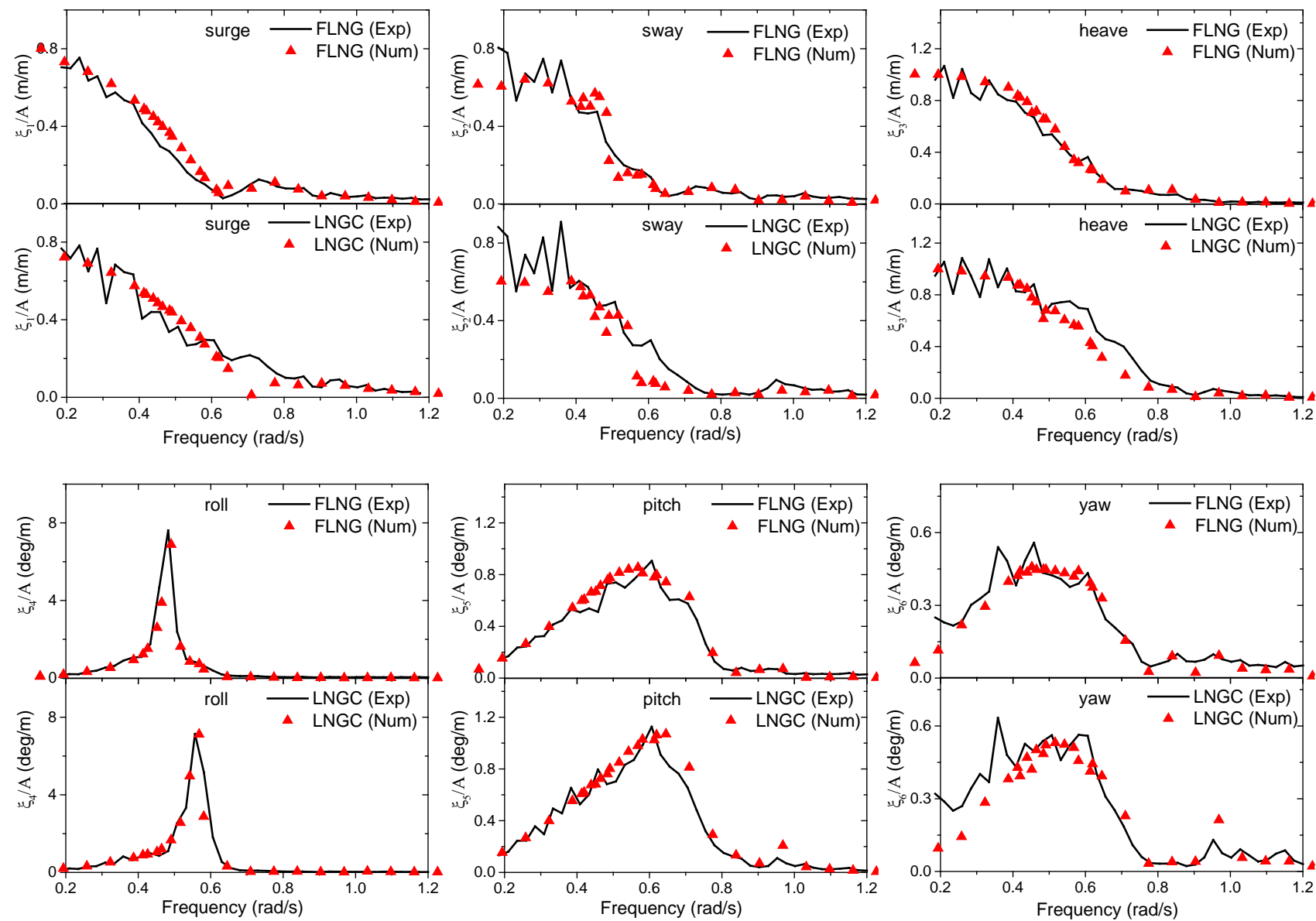

Fig. 15 RAOs of vessels’ motions under quarter wave (135 degree) 

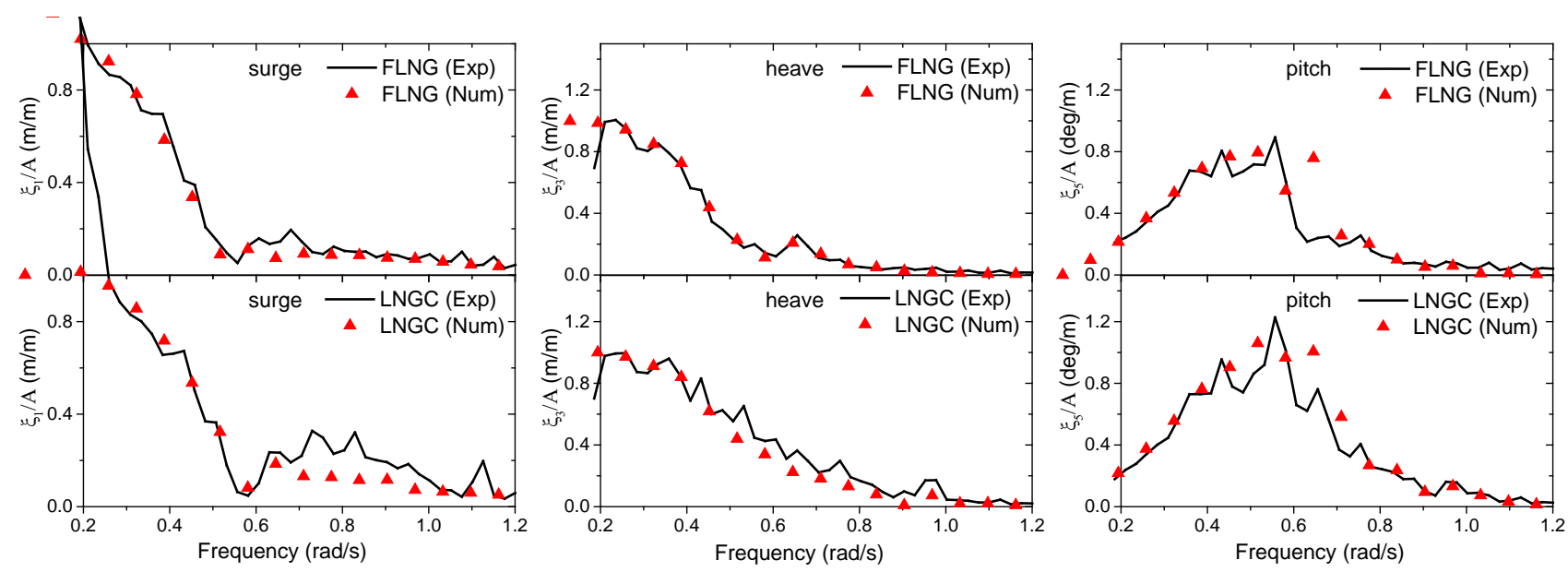

Fig. 16 RAOs of vessels' motions under heading wave

\section{Discussion}

In this section, dynamic responses of the FLNG system are investigated based on numerical and experimental results. Firstly, hydrodynamic interactions between vessels under different wave directions, sloshing effects on vessels' motions and loads on connection system are analyzed without considering the connection system for the convergence of analysis. Then, responses of connection system including sloshing effects are studied.

\subsection{Hydrodynamic interaction between vessels}

For side-by-side arranged FLNG vessel and LNG carrier, the existence of the adjacent vessel can change the boundary condition in the fluid field, which will affect the hydrodynamic coefficients of vessels and wave forces on vessels. In the single vessel case, the radiation wave can transmit to infinity and disappear; in the side-by-side arranged vessels, the radiation wave of the moving vessel will be reflected by the adjacent vessel and radiation energy dissipates slowly. Roll motion retardation function are compared for FLNG vessel in single vessel condition and side-by-side configuration. As shown in Fig. 17, retardation function of single vessel case decays much faster than that in side-by-side configuration. As retardation function will decay to zeros gradually, convolution calculation in equation (11) form the beginning $(t=0)$ is not always necessary. But the calculation should include relatively longer time in side-by-side conditions than in single vessel conditions.

Hydrodynamic interactions between vessels are sensitive to the wave direction. Fig. 18 shows the motion responses of vessels under two beam sea conditions without consideration of connection system. FLNG vessel locates in the weather side and lee side in 90 degree and 270 degree headings, respectively. It can be seen that vessel motions tend to have larger responses when the vessel is located in the weather side. For sway and heave motions, sheltering effects mainly lies in the high frequency region; for roll motion, a slight response amplitude decrease appears in the natural roll frequency when vessels are located in lee side. In addition, no obvious responses are induced near the frequency of gap resonance, which indicates the resonance in the gap has less effect on vessels' motions.

The effects of radiation wave caused by the motions of the adjacent vessel are investigated. RAOs of the vessels' motion under beam sea (90 degree) are plotted in Fig. 19. The differences between the two cases with adjacent vessel free floating and fixed reflect the radiation wave effects. It can be seen that the RAOs of vessel's motions are much smaller when the adjacent vessel is fixed, especially in the sway and have modes, which indicate the radiation caused by the vessel's motions can amplify the adjacent vessel's motion responses significantly. In the roll mode, when FLNG is fixed, the roll motion of LNG carrier is much small as the FLNG is located in the 
weather side and wave excitation on LNG carrier decreases for the shielding effects, and the free floating FLNG can excite much larger roll motion of LNG carrier. The RAOs of FLNG's roll motions show the radiation wave produced by the roll motion of LNG carrier contributes less to the roll motion of FLNG. The reason could be that the roll motion of LNG carrier is decreased for the shielding effects, besides, the differences in displacement also makes LNG carrier more sensitive to the motion of adjacent vessel than FLNG.

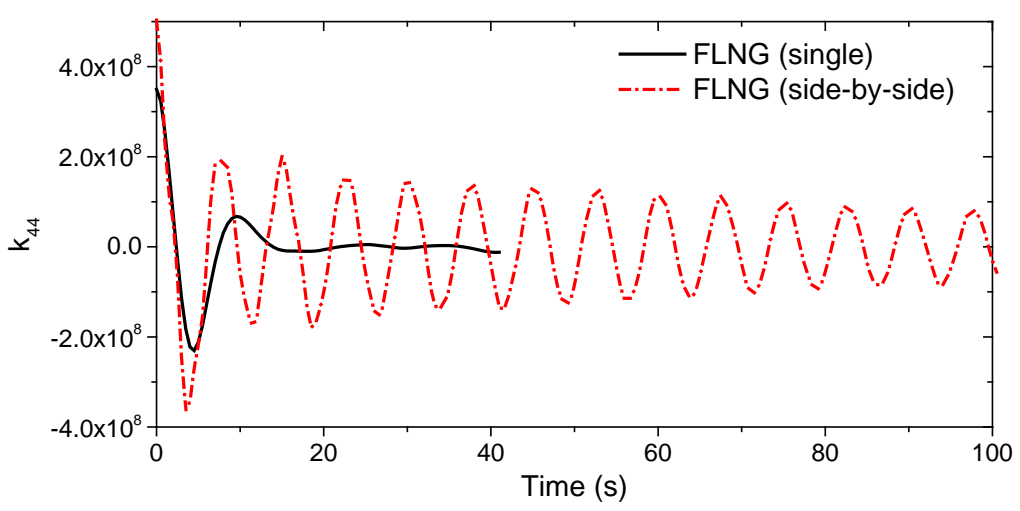

Fig. 17 Retardation function of FLNG in roll mode
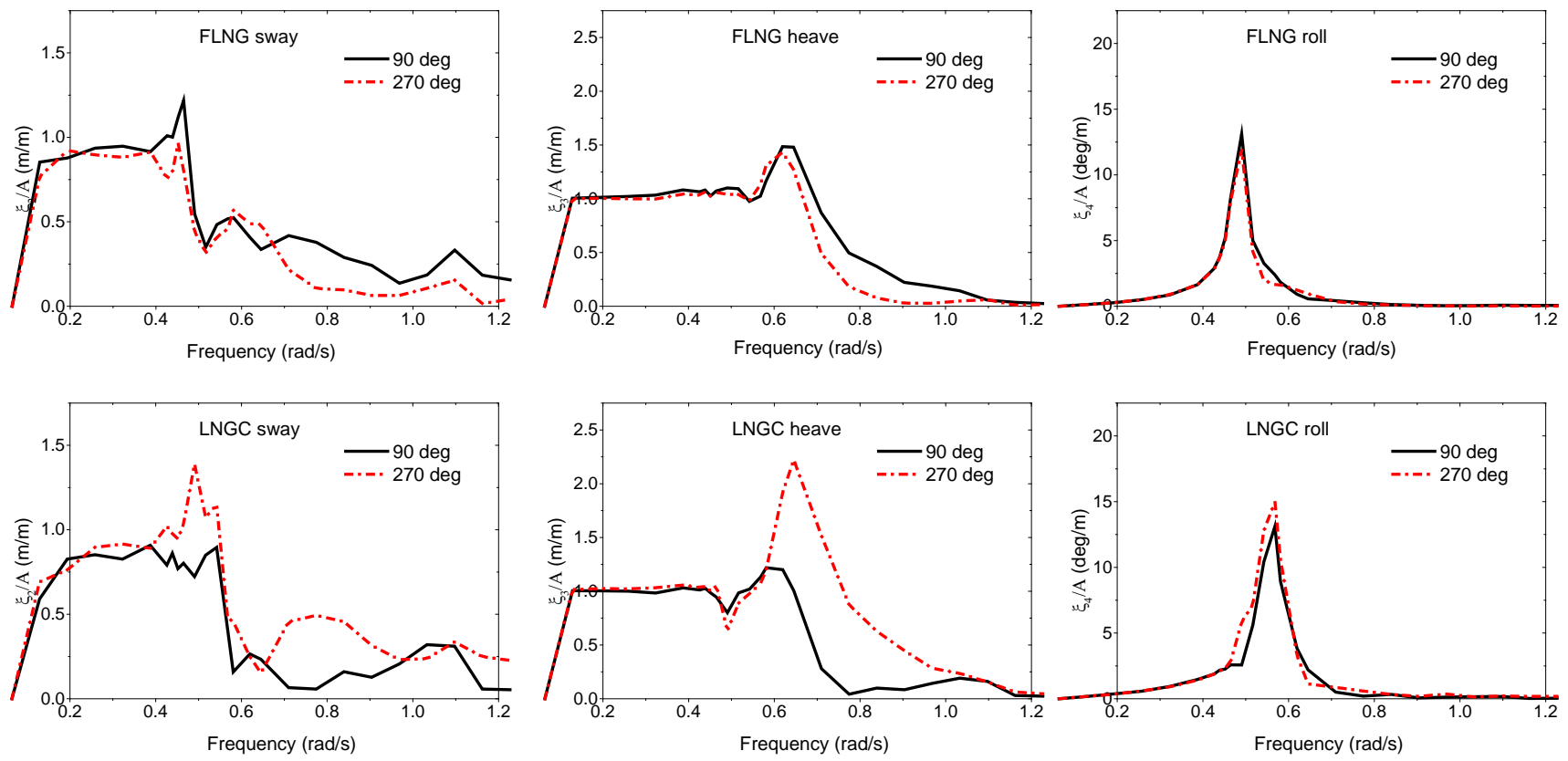

Fig. 18 Numerical RAO results of vessels’ motions under beam sea conditions 

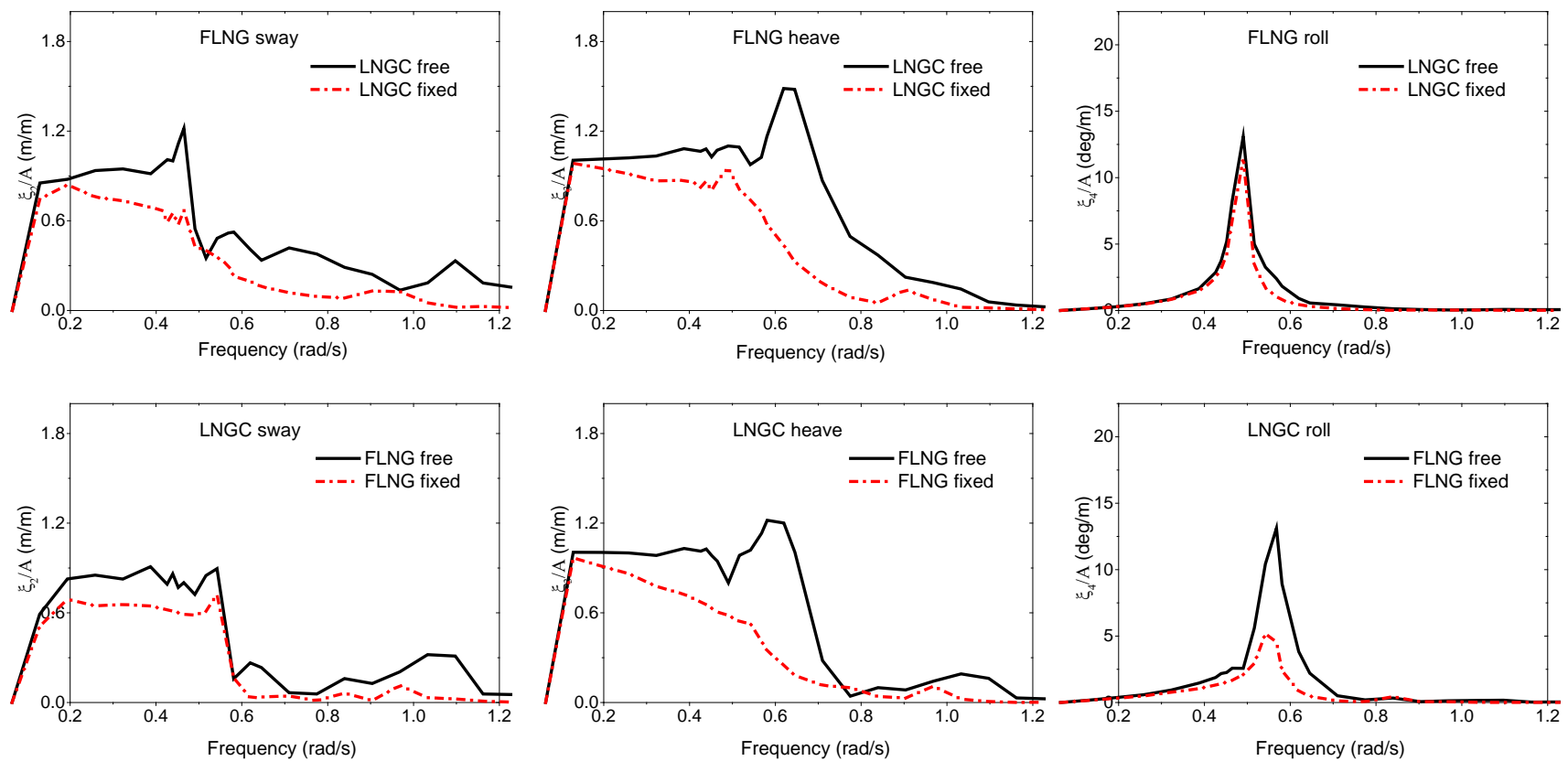

Fig. 19 Numerical RAO results of vessels’ motions without radiation potential under beam sea condition (90 degree)

\subsection{Sloshing effects}

Sloshing effects on the responses of FLNG system are investigated in this section. Damping coefficient $\mu=0.1$ is adopted in the artificial damping model that described in 2.2. Vessels' motion and sloshing loads on vessels are discussed. Sloshing effects in different filling levels are compared and nonlinear effects are also considered.

\section{Liquid loading effects}

Comparisons of motion responses in solid and liquid loading conditions under beam sea are conducted. The FLNG and LNG carrier are $66.7 \%$ and $60 \%$ filled, respectively; the corresponding first mode natural sloshing frequencies of liquid tanks in FLNG and LNG carrier are $0.886 \mathrm{rad} / \mathrm{s}$ and $1.018 \mathrm{rad} / \mathrm{s}$, respectively. Sway, heave and roll motions of vessels are presented in Fig. 20. It is indicated that numerical results have good agreements with experimental results in both solid and liquid loading conditions. The sway motions of vessels in liquid loading condition have small response amplitudes in the natural sloshing frequency and larger responses in the frequency region higher than the natural sloshing frequency. The heave motions of vessels are less affected by liquid sloshing. The roll motions of vessels in liquid loading conditions have smaller natural frequencies and response amplitudes than those in solid loading conditions. The natural roll motion frequencies of FLNG and LNG carrier decrease from $0.48 \mathrm{rad} / \mathrm{s}$ and $0.58 \mathrm{rad} / \mathrm{s}$ in the solid loading conditions to $0.42 \mathrm{rad} / \mathrm{s}$ and $0.52 \mathrm{rad} / \mathrm{s}$ in the liquid loading conditions, respectively. Fig. 21 shows the RAOs of sloshing wave elevations and sloshing loads in the sway and roll modes. Two peaks corresponding to natural roll motion frequency and natural sloshing frequency can be observed from sloshing elevations. It can be pointed out that the two peaks of sloshing elevation exert larger sloshing loads in roll mode and sway mode, respectively. As revealed in Section 4.2, the two sloshing peaks around the natural roll motion frequencies and natural sloshing frequencies are mainly excited by the vessels' roll motion and sway motion, respectively.

Responses of liquid loading vessels under 270 degree beam sea condition are presented in Fig. 22 for comparison. As solid loading cases shown in Fig. 18, for the shielding effects, the vessel tends to have larger heave and sway motion responses when it is located in the weather side. But this tendency cannot be found from the roll 
motions of vessels in liquid loading condition. The RAOs of roll motion of the vessels have slight differences with the change of wave direction, which indicates the sloshing effects on vessels' roll motion are much significant than the interaction effects between vessels under different wave directions. Phase shifts of sloshing loads to wave forces are presented in Fig. 23 to investigate the sloshing contributions to the motions of vessels. The phase shifts in sway and roll modes have similar distributions except in the frequency regions lower than the natural roll motion frequencies. The phase shifts are also affected by the wave directions. According to the phase shifts in roll mode, for the vessels located in the weather side, the sloshing loads contribute to roll motions positively in the frequency region smaller than the natural roll frequency and negatively in the frequency region between natural roll frequencies and the natural sloshing frequencies. The phase shifts change from positive to negative in the natural sloshing frequencies. For the vessels in the lee side, the phase shifts in the frequency regions between the natural roll frequencies and the natural sloshing frequencies are smaller than those of vessels located in the weather side, and the phase shifts change from positive to negative in the natural roll motion frequencies. Moreover, in the frequency regions higher than natural sloshing frequencies, phase shifts are much smaller except for LNG carrier located in lee side. As significant sloshing loads in sway mode are excited in the frequency regions higher than the natural sloshing frequencies, the RAO peaks of sway motion in these regions can be explained.
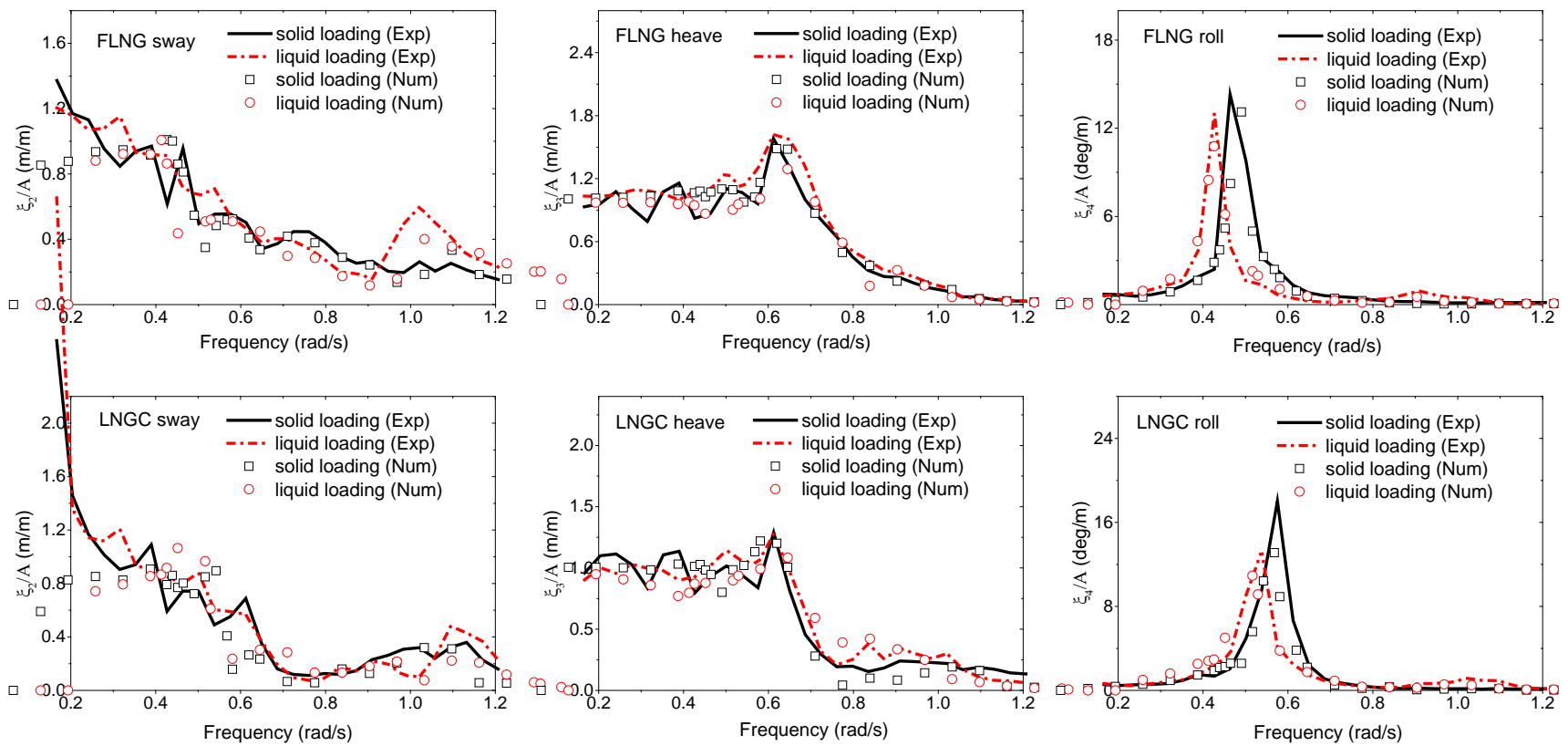

Fig. 20 Numerical RAO results of vessels’ motions in solid and liquid loadings under beam sea condition (90 deg)
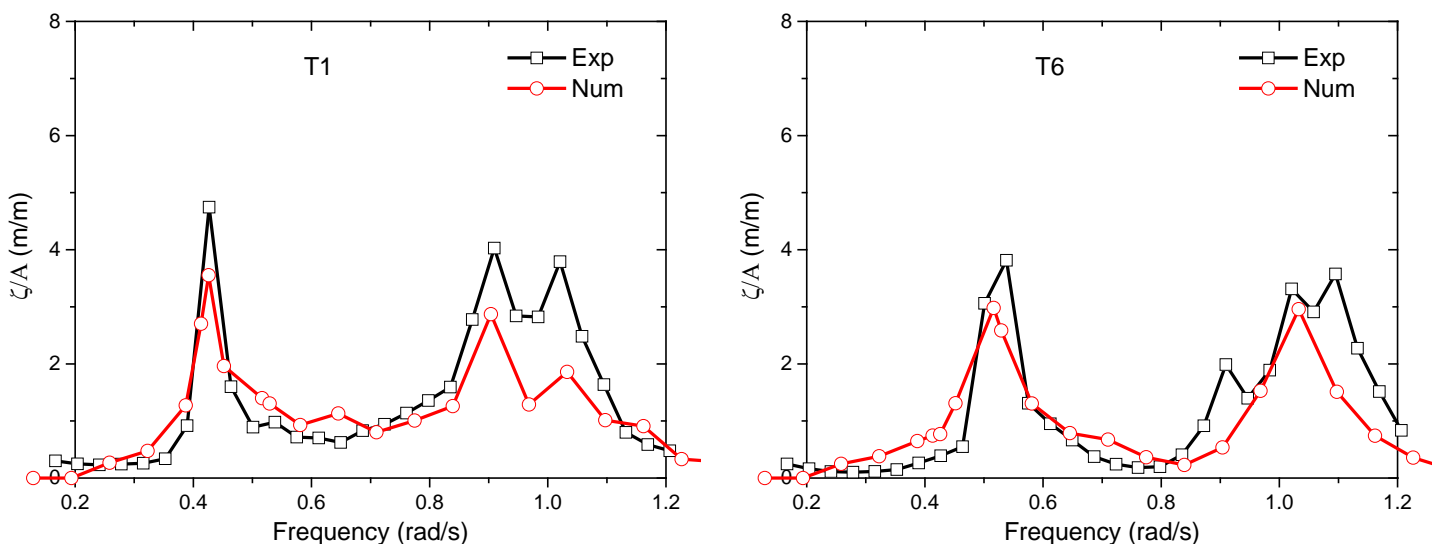

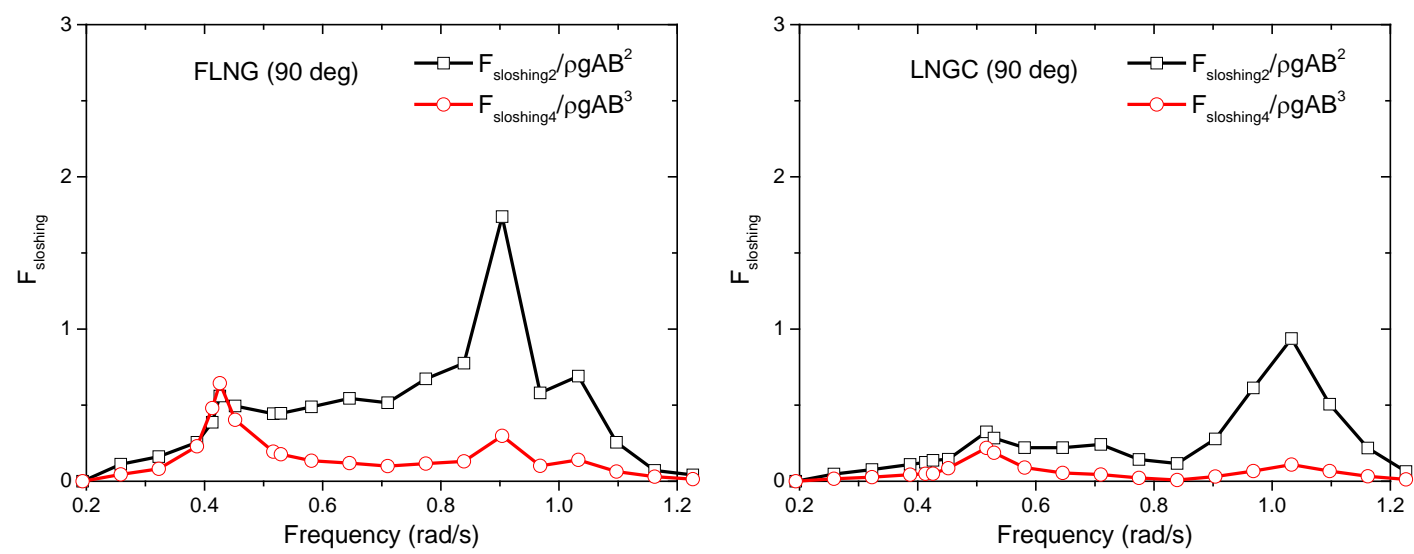

Fig. 21 Numerical RAO results of sloshing elevations and sloshing loads under beam sea condition (90 deg)
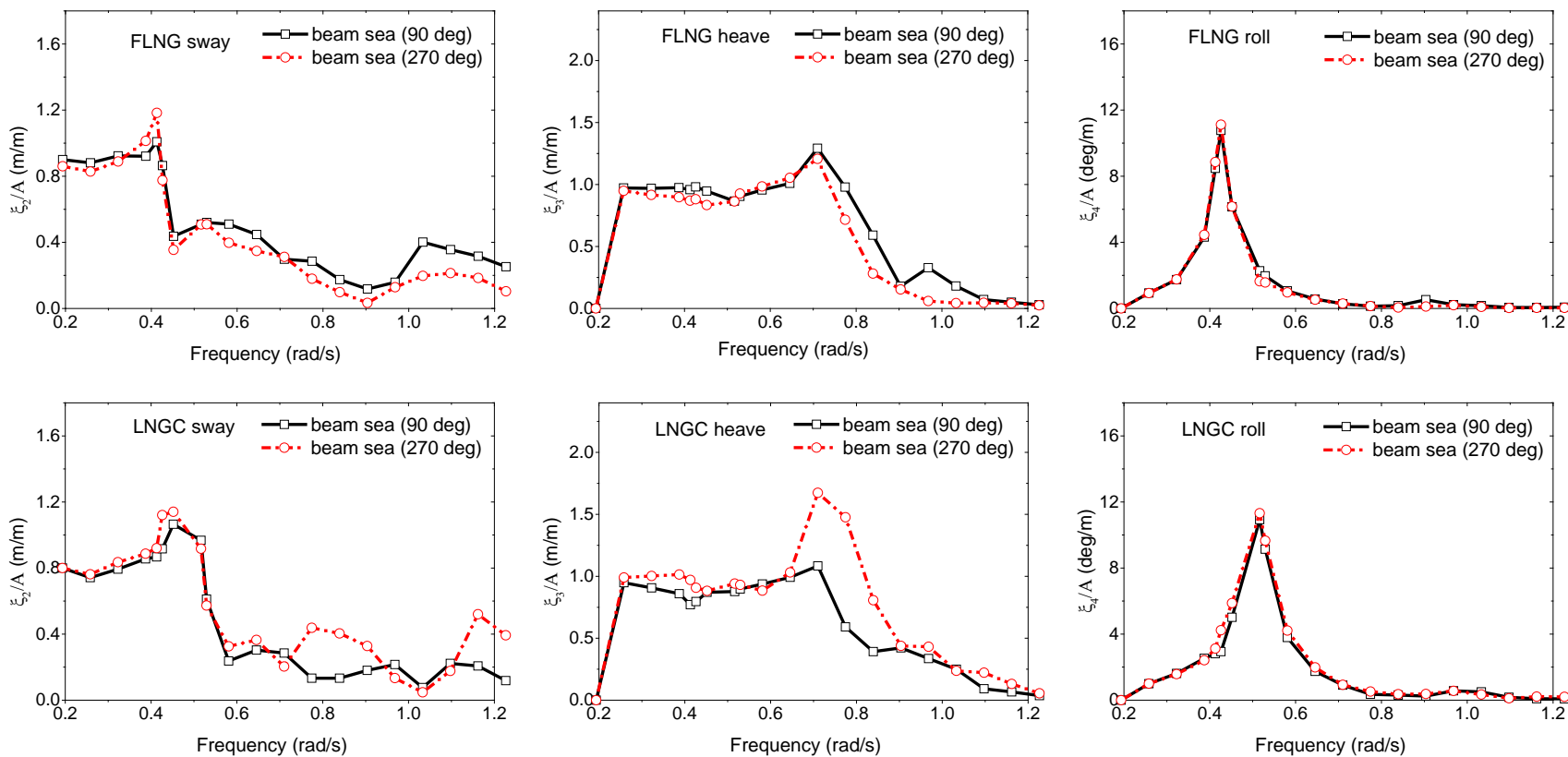

Fig. 22 Numerical RAO results of vessels' motions in liquid loading under beam sea conditions
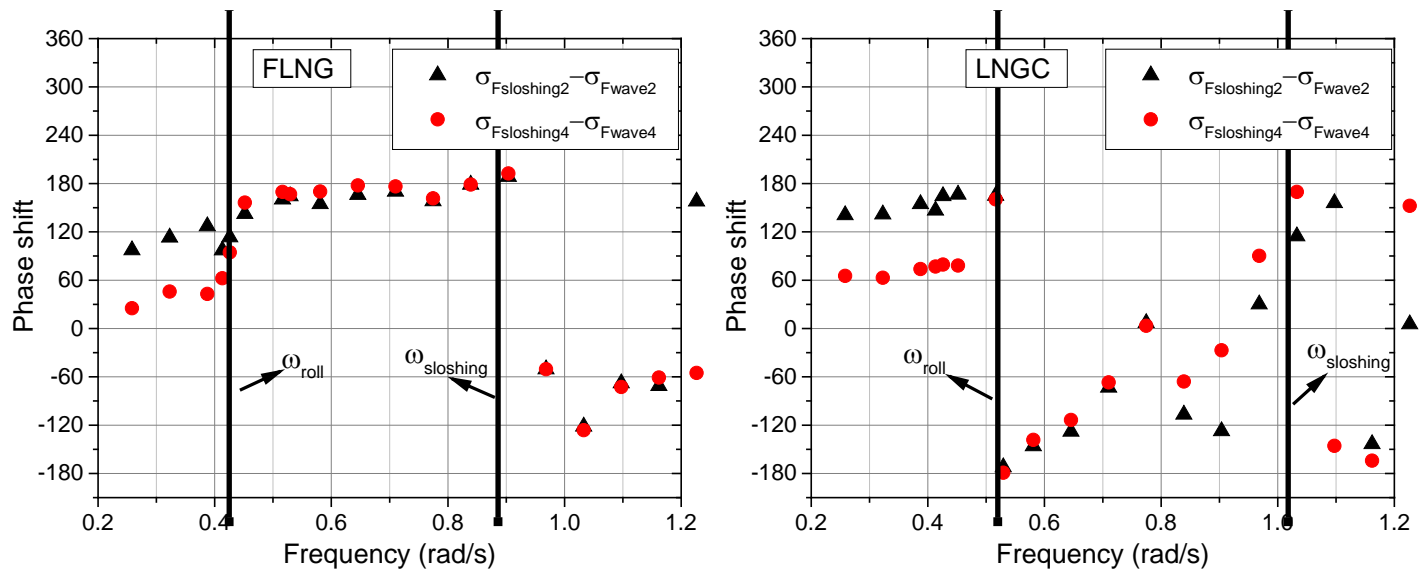

beam sea (90deg) 

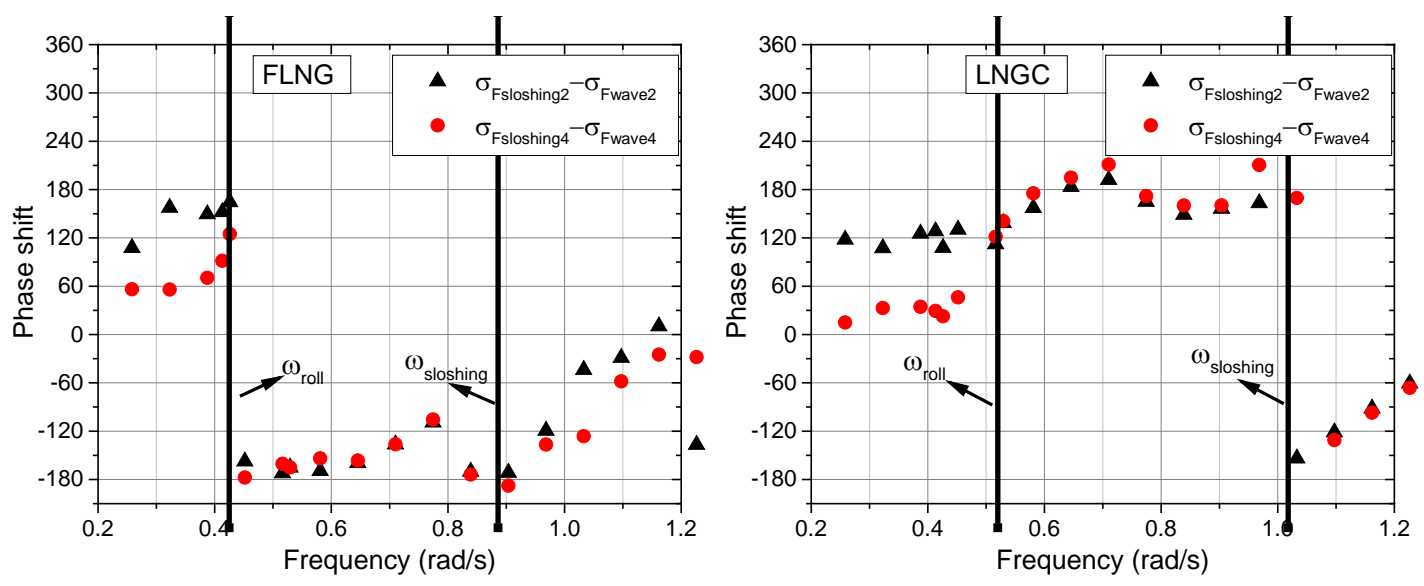

beam sea (270deg)

Fig. 23 Numerical phase shifts between wave forces and sloshing loads for vessels in liquid loading condition

\section{Filling level effects}

Responses of the FLNG system in different filling levels are discussed here. Four fill cases are selected, as shown in Table 4. Each vessel has two filling levels. First mode sloshing frequencies in liquid tanks are presented.

The RAOs of the vessels' motions in different filling levels are shown in Fig. 24. The sway motions tend to have larger responses in the low filling levels, especially for LNG carrier. The reason is the low filling level tanks have smaller natural sloshing frequencies and larger sloshing can be excited when the natural sloshing frequencies are close to the main response frequency regions of vessels' motions. As shown in Fig. 25, low filling level cases have larger sloshing responses in the frequency region between the natural roll frequency and natural sloshing frequency. The natural frequencies of FLNG's roll motion vary with the change of filling levels. Compared with solid loading condition, the natural roll motion frequency of FLNG decreases to $0.42 \mathrm{rad} / \mathrm{s}$ in $66.7 \%$ filling level and increases to $0.52 \mathrm{rad} / \mathrm{s}$ in $33.3 \%$ filling level; the natural roll motion frequency of LNG carrier decreases from $0.58 \mathrm{rad} / \mathrm{s}$ in solid loading condition to $0.52 \mathrm{rad} / \mathrm{s}$ in $60 \%$ filling level and increases to $0.61 \mathrm{rad} / \mathrm{s}$ in $24 \%$ filling level. It can be seen that natural roll frequencies tend to be larger than solid loading conditions in low fill condition and smaller than solid loading conditions in high filling level. Fig. 25 shows sloshing loads in roll mode are much small in the low fill conditions, because sloshing loads in roll mode are mainly caused by static pressure related to the liquid mass rather than dynamic pressure as in sway mode.

The sloshing in liquid tanks can also affect the hydrodynamic interaction between vessels. The motion and sloshing responses of FLNG in same filling level have slight change with the variance of the filling level of LNG carrier, while the variance of filling level of FLNG can cause different responses of LNG carrier in the same filling levels. As shown in Fig. 24, with different filling levels of the FLNG, LNG carrier has different sway motions in fill Cases B and D and has different roll motions in Case A and Case C, which correspond to the conclusion drawn above that sloshing has more effects on the sway motion in the low filling level conditions and has more effects on the roll motion in the high filling level conditions for the large liquid mass. As vessels in different filling levels have the same inertia when liquid in tanks is frozen, this kind of interaction can be regarded as the change of radiation effects caused by liquid sloshing on the adjacent vessel. The effects of FLNG's motions on LNG carrier are more obvious for their differences in displacement.

Table 4 Natural sloshing frequencies in liquid tanks under different filling levels

\begin{tabular}{c|cc|cc|cc|cc}
\hline Fill case & \multicolumn{2}{|c|}{ A } & \multicolumn{2}{c|}{ B } & \multicolumn{2}{c|}{ C } & \multicolumn{2}{c}{ D } \\
\hline & FLNG & $\begin{array}{c}\text { LNG } \\
\text { carrier }\end{array}$ & FLNG & $\begin{array}{c}\text { LNG } \\
\text { carrier }\end{array}$ & FLNG & $\begin{array}{c}\text { LNG } \\
\text { carrier }\end{array}$ & FLNG & $\begin{array}{c}\text { LNG } \\
\text { carrier }\end{array}$ \\
\hline Filling level & $66.7 \%$ & $60 \%$ & $66.7 \%$ & $25 \%$ & $33.3 \%$ & $60 \%$ & $33.3 \%$ & $25 \%$ \\
\hline
\end{tabular}




\begin{tabular}{c|cc|cc|cc|cc}
\hline $\begin{array}{c}\text { Natural frequency } \\
(\mathrm{rad} / \mathrm{s})\end{array}$ & 0.886 & 1.018 & 0.886 & 0.814 & 0.749 & 1.018 & 0.749 & 0.814 \\
\hline
\end{tabular}
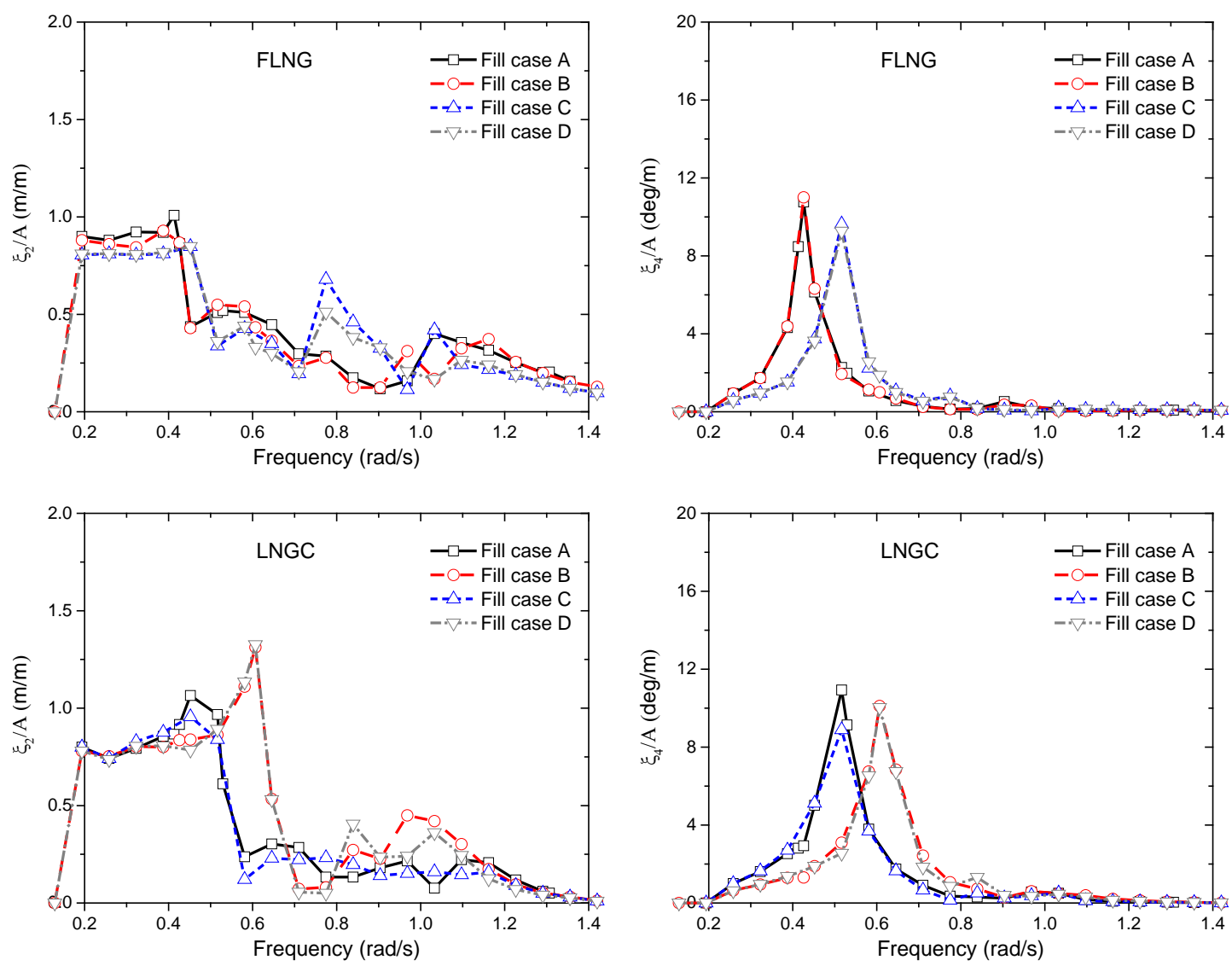

Fig. 24 Numerical RAO results of vessels' motions in different filling levels
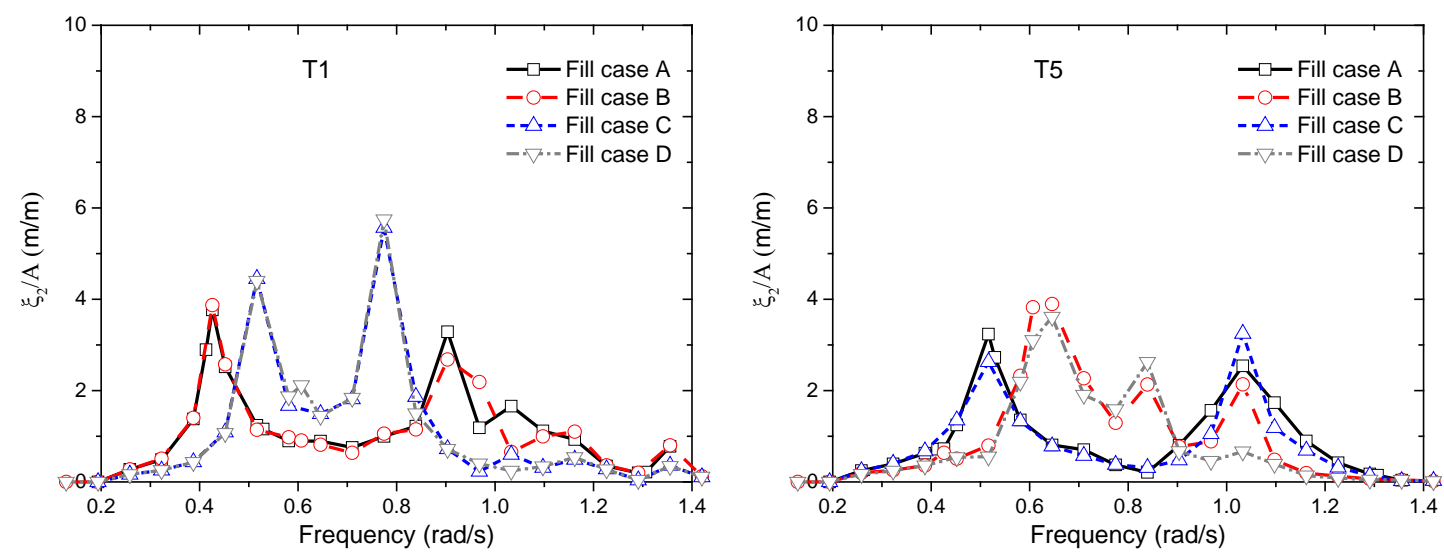

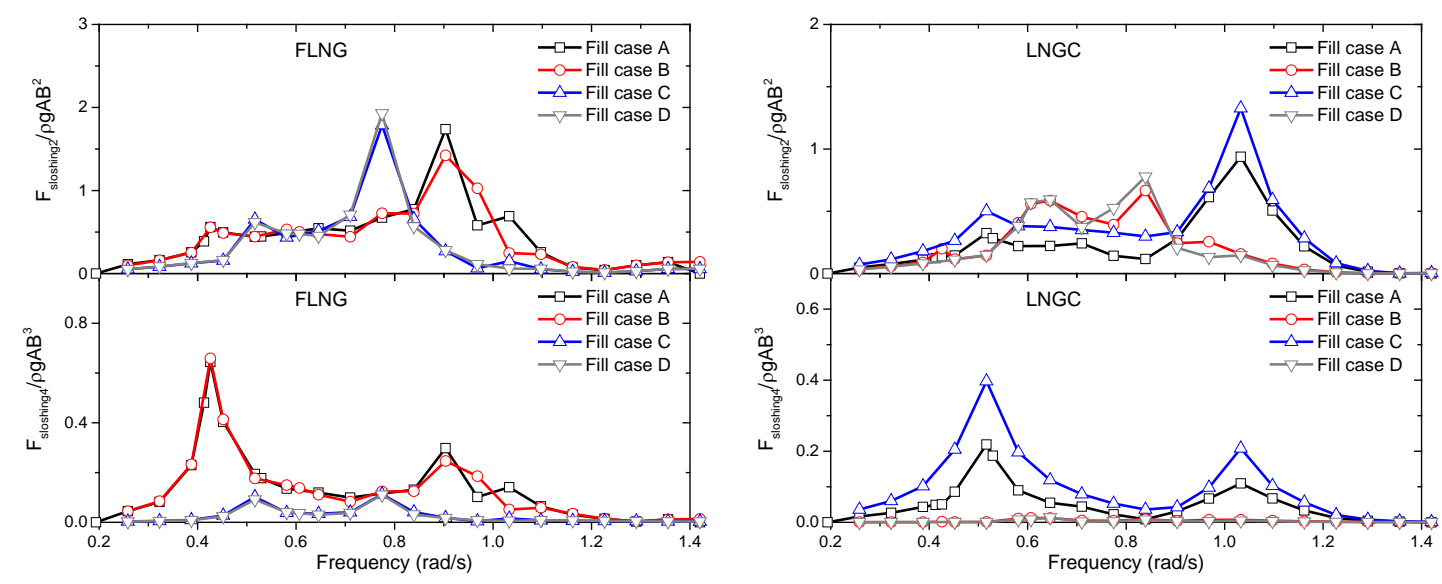

Fig. 25 Numerical RAO results of sloshing elevations and loads on vessels in different filling levels

\section{Sloshing nonlinearity}

Nonlinear free surface is adopted in the sloshing calculation, and the nonlinearity of sloshing will become obvious with the increase of wave amplitude, especially for the shallow filling level conditions. Fill Case D is selected and a smaller wave amplitude equals 0.5A is added. Fig. 26 shows the RAOs of the vessels' motions under different wave amplitudes, the motion RAOs are slightly changed with the increase of wave amplitude. The wave elevation histories in T1 and T6 under regular waves are plotted in Fig. 27. Regular wave frequencies (0.52, 0.61, 0.77, $0.84 \mathrm{rad} / \mathrm{s}$ ) around the natural roll motion frequencies and sloshing frequencies of vessels in the fill Case D are selected. It can be seen sloshing wave elevation in the natural roll frequencies has no obvious nonlinearity, while sloshing near the natural sloshing frequencies demonstrate obvious nonlinear properties with the increase of wave amplitude. As discussed above, sloshing around the natural roll frequencies are mainly excited by tank inclination and is linearly related to vessels' roll motion; while sloshing around the natural sloshing frequencies are dynamically excited by sway motion and has nonlinear relationships with excitation amplitude. Fig. 29 show the free surface profiles in the LNG carrier liquid tank at the maximum wave elevation. The free surface is much flat in the case $0.61 \mathrm{rad} / \mathrm{s}$ as the sloshing is caused by the inclination of tank related to the LNG carrier's roll motion. In the case $0.84 \mathrm{rad} / \mathrm{s}$, the free surface demonstrates first order mode and obvious nonlinearity can be observed. But the sloshing nonlinearity does not change the sloshing loads exerted on vessels much, as shown in Fig. 28. The reason is that sloshing loads on tanks are decided by the differences of wave elevation in the two sides of tank, the nonlinear sloshing with higher crest and lower trough contributes less to the total loads except in the sloshing cases with strong nonlinearities. Thus, the RAOs of liquid loading vessels' motions shown in Fig. 26 do not demonstrate nonlinear properties under increased wave amplitude. When the wave amplitude continues to increase, the sloshing will become much violent and wave breaking will occur, which beyond the limitation of the potential flow theory. Research conducted by Jiang et al. (2015) shows smaller roll motion RAOs are induced when the sloshing gets violent. 

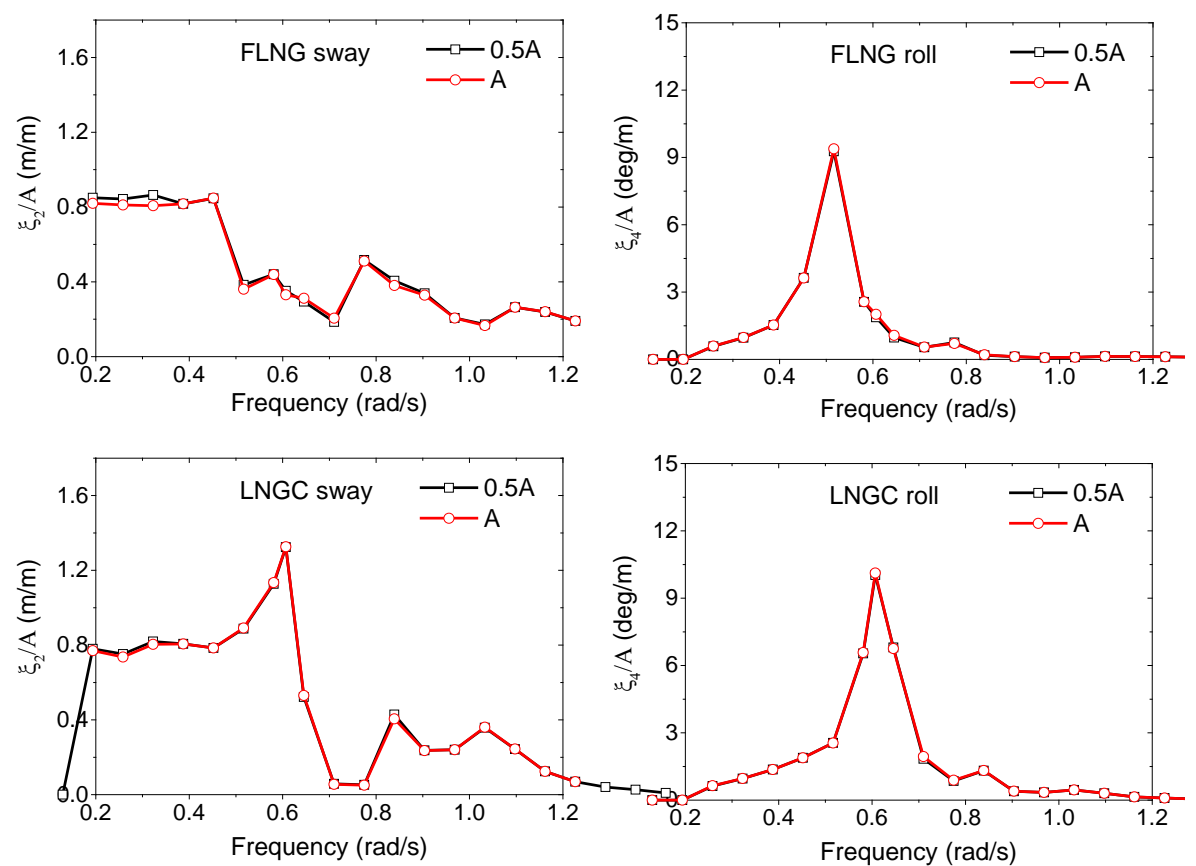

Fig. 26 Responses of vessels under different wave amplitudes
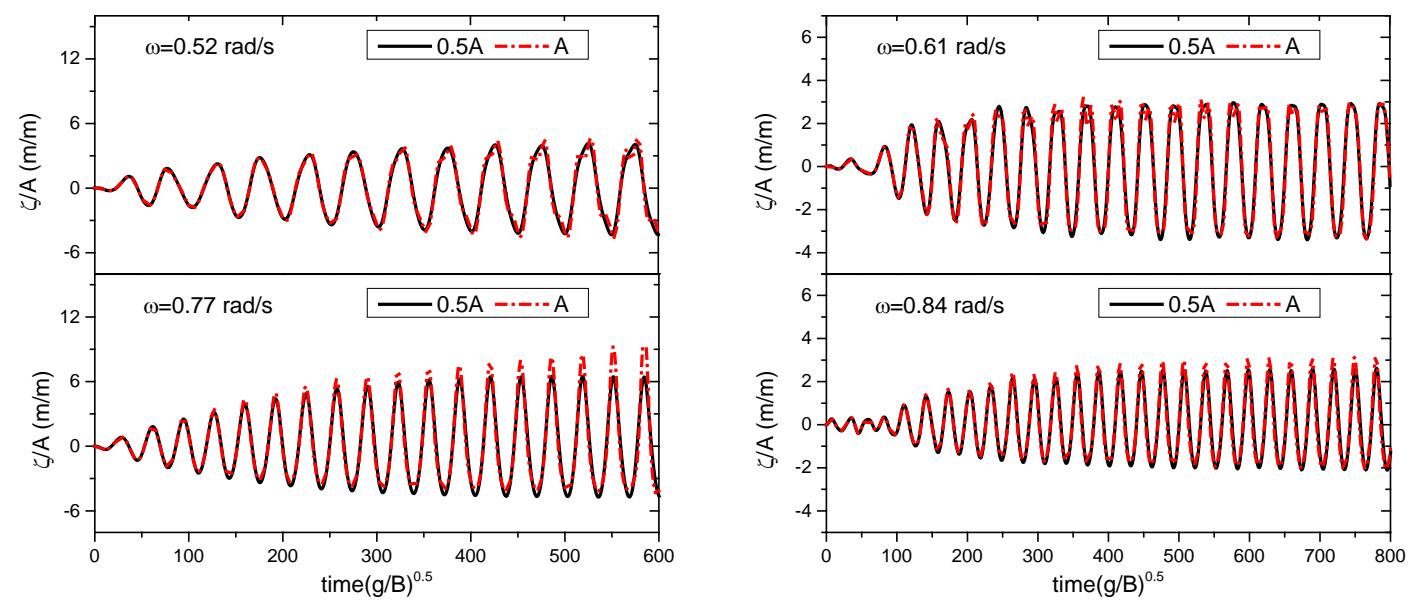

Fig. 27 Histories of sloshing elevations
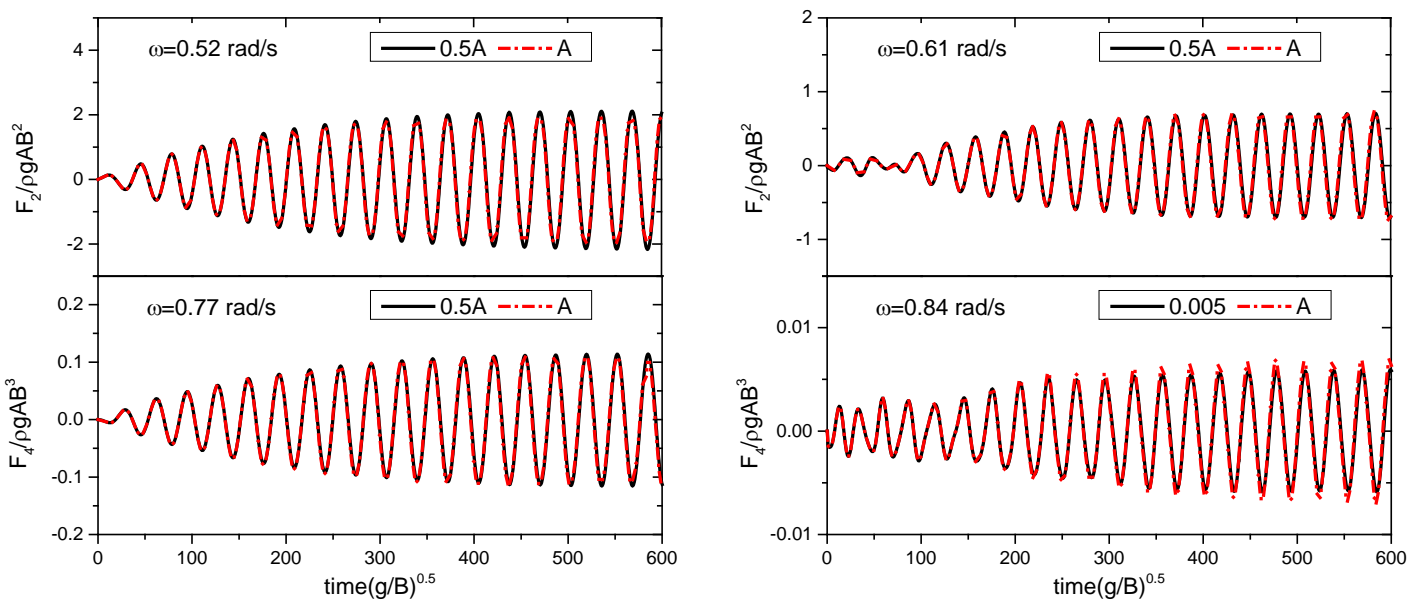

Fig. 28 Histories of sloshing loads 


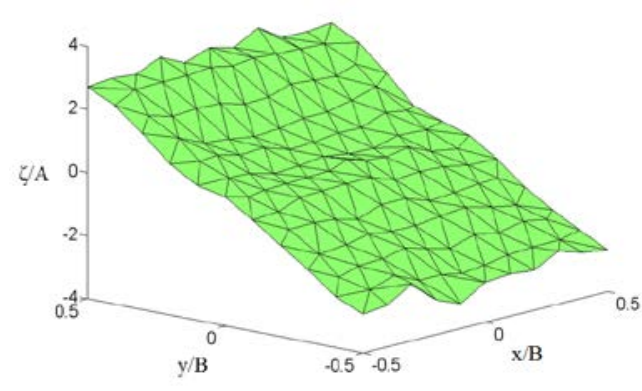

$0.61 \mathrm{rad} / \mathrm{s}$

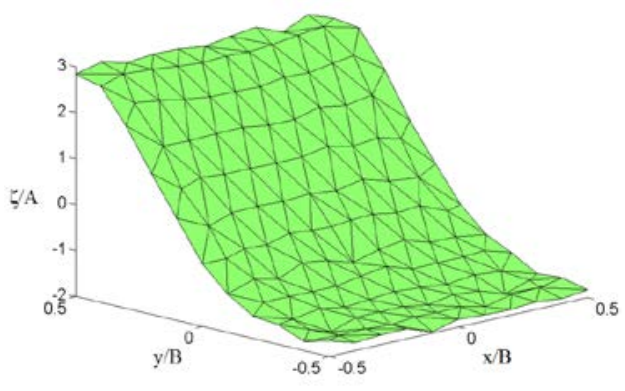

$0.84 \mathrm{rad} / \mathrm{s}$

Fig. 29 Free surface of sloshing in the liquid tank of LNG carrier

\subsection{Connection system responses}

Responses of the FLNG system with connection system are discussed in this section. Restriction effects of the connection system on the vessels' motion and loads on connection system are analyzed. FLNG system is restricted with soft horizontal spring lines rather than single-mooring system in this study. Thus, weather-vaning property will not occur as in practice and loads on connection system are mainly related to the wave excited motions of vessels.

The responses of FLNG system with connection system in solid loading condition are considered. The motion RAOs of the vessels under quarter (135 degree) wave are presented in Fig. 30, the numerical results have good agreements with experimental results. It can be seen that the surge motions have large value in $0.39 \mathrm{rad} / \mathrm{s}$. RAOs of the loads on hawsers the relative motions between the two vessels are shown in Fig. 31 and 32, respectively. Corresponding to peaks in the surge motions, peaks values of loads on hawsers and relative motions in sway mode also appear in $0.39 \mathrm{rad} / \mathrm{s}$. It can be deduced that the resonant responses of connection system in sway mode are excited. For the FLNG system consists of two floating vessels connected with springs, natural frequency of the connection system can be calculated as:

$$
\omega=\sqrt{\frac{K_{\text {connect }}\left(M_{1}+M_{2}\right)}{M_{1} M_{2}}}
$$

where $K_{\text {connect }}$ stands for stiffness of connection system in specific direction, $M_{1}$ and $M_{2}$ are the mass (including added mass) of FLNG and LNG carrier, respectively. Resonant responses will occur when the natural frequency of connection system meet with wave frequency. Relative motions in other modes are not as obvious as in surge mode; the two peaks in the relative roll motion respectively correspond to the natural roll frequencies of FLNG and LNG carrier. Moreover, it can be observed from experimental results that LNG carrier has larger sway and yaw motions, which may be caused by the second wave forces and cannot be found in the numerical results.

Sensitivities to the stiffness of the connection system are checked to optimize the motion responses of the vessels. Fig. 33 shows the RAOs of vessels' motions with different connection system stiffness defined based on $K$ shown in Table 2. With the stiffness of the connection system decreased to $K_{\text {connect }}=0.25 K$, resonant frequency in surge mode reduces to half with much smaller peak response. In the case $K_{\text {connect }}=0.1 K$, the natural frequency of connection is much small and no resonant responses are induced. The motions of vessels are slightly affected by the connection system for the small stiffness. When connection system has large stiffness $K_{\text {connect }}=10 \mathrm{~K}$, motions in surge, heave and roll modes are decreased significantly. The reason could be that the two vessels are connected more closely for the larger stiffness and motions of vessel are more affected by the adjacent vessel through the connection system. For the large stiffness, the roll motion responses of FLNG and LNG carrier have same natural roll frequency, which is larger than the natural roll frequency of the FLNG and smaller than the natural roll 
frequency of the LNG carrier in the case without connection system. Moreover, sway and yaw motions have increased responses in the case $K_{\text {connect }}=10 \mathrm{~K}$. The reason could be that the resonance of the connection system in sway mode is excited. Fig. 34 shows the loads on the hawsers with different stiffness of the connection system. With the increase of the stiffness, the loads on the hawsers increase correspondingly.

Sloshing effects on the connection system are analyzed by comparing responses of FLNG system in solid and liquid loading conditions. Stiffness of the connection system is set as $K_{\text {connect }}=0.1 K$. The RAOs of vessels' motions and loads on the hawsers are presented Fig s. 35 and 36. As demonstrated in Section 5.1, for liquid loading condition, larger surge motions are induced by sloshing loads in the frequency region higher than natural sloshing frequency, and larger loads on hawsers can be found in liquid loading condition from Fig. 36. Roll motions have decreased natural roll frequency in the liquid loading condition. As sway motion couples closely with roll motions, obvious frequency shift between solid and liquid loading conditions in sway motion RAO can be observed. As a result, the peak value of loads on Hawser 1 appears in a lower frequency. Vessels in liquid loading condition also have smaller pitch motion RAOs, while the response amplitudes are rather small and its influences on hawsers are not obvious.

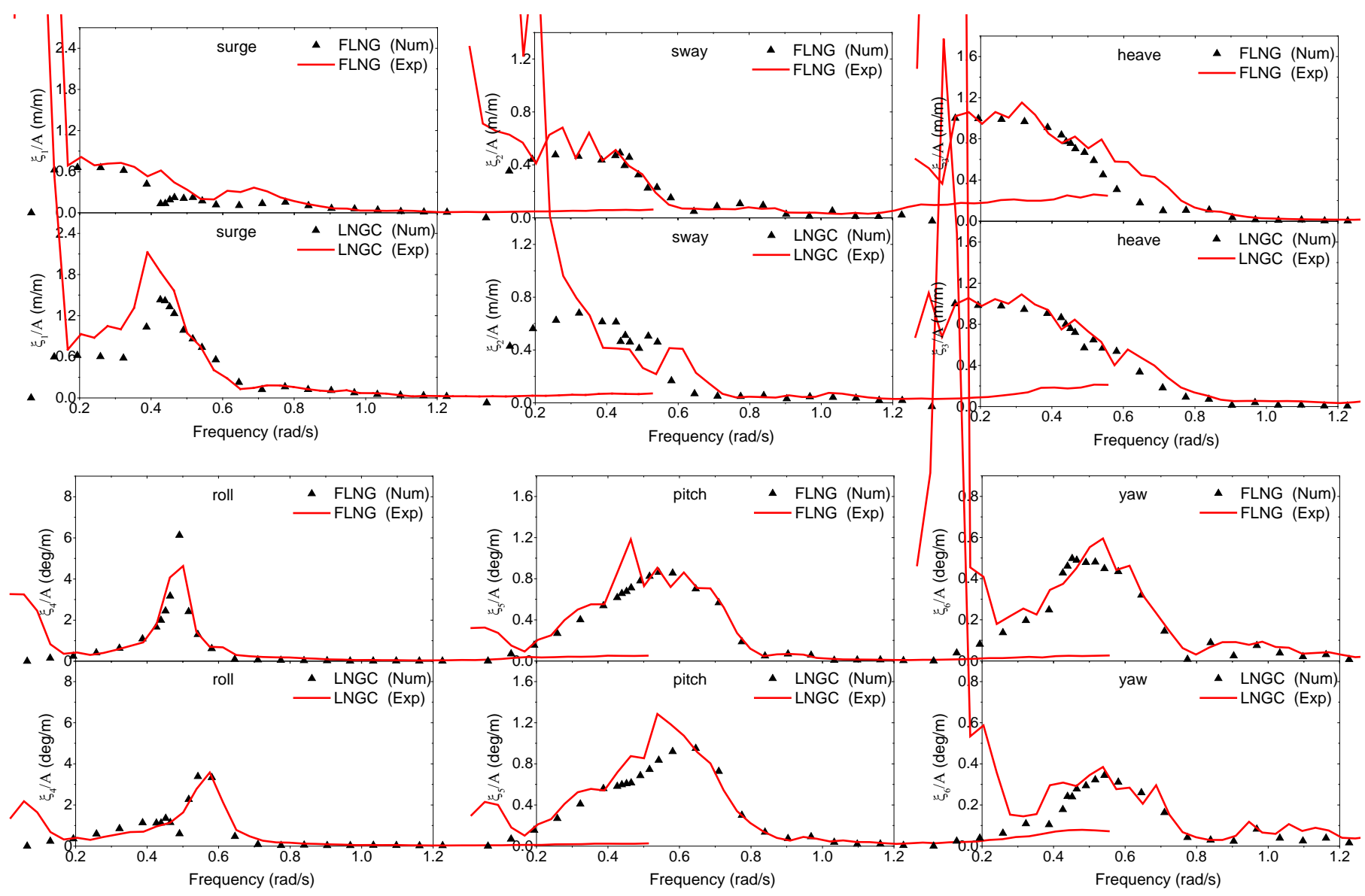

Fig. 30 RAOs of vessels' motion with connection system 


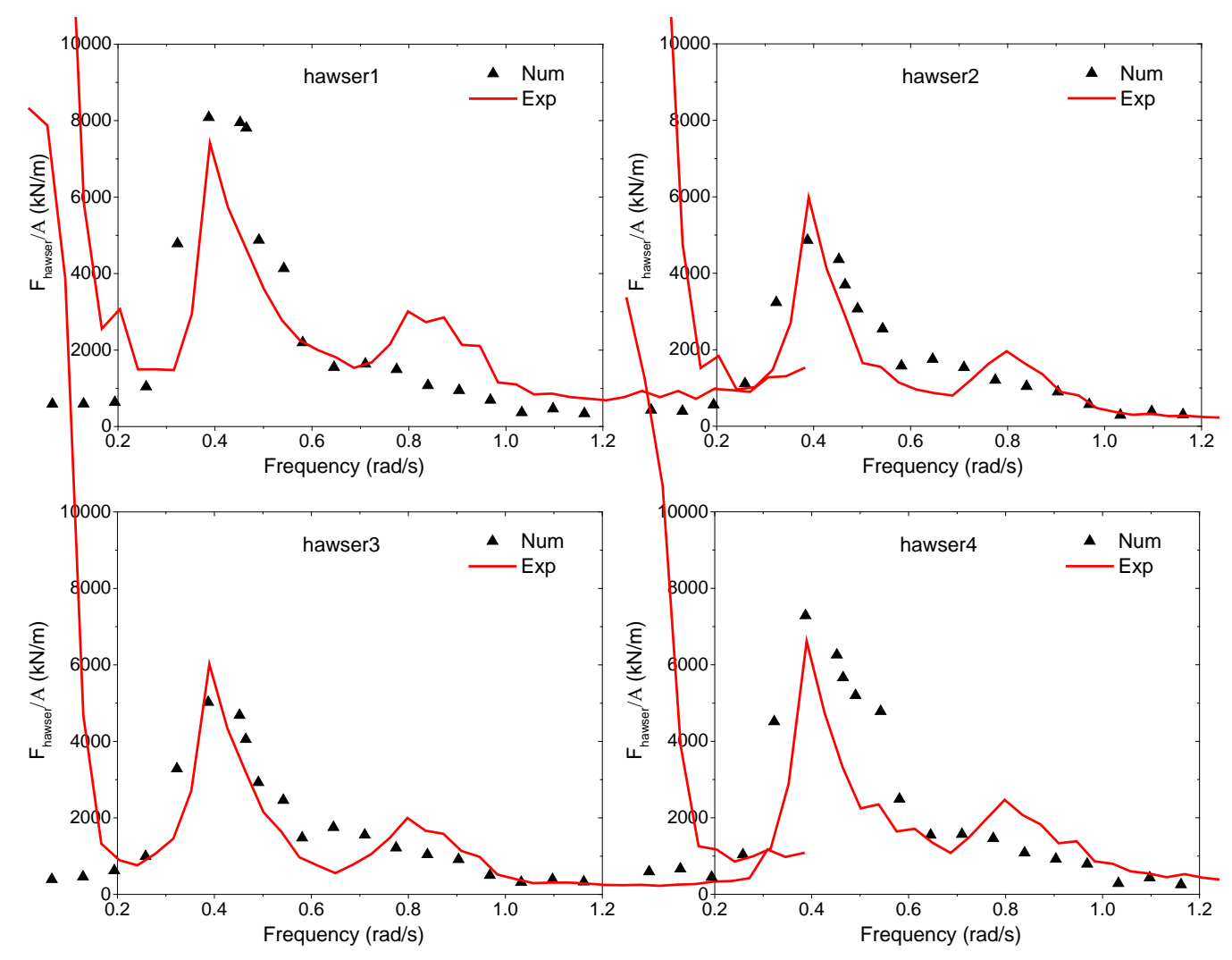

Fig. 31 RAOs of loads on hawsers
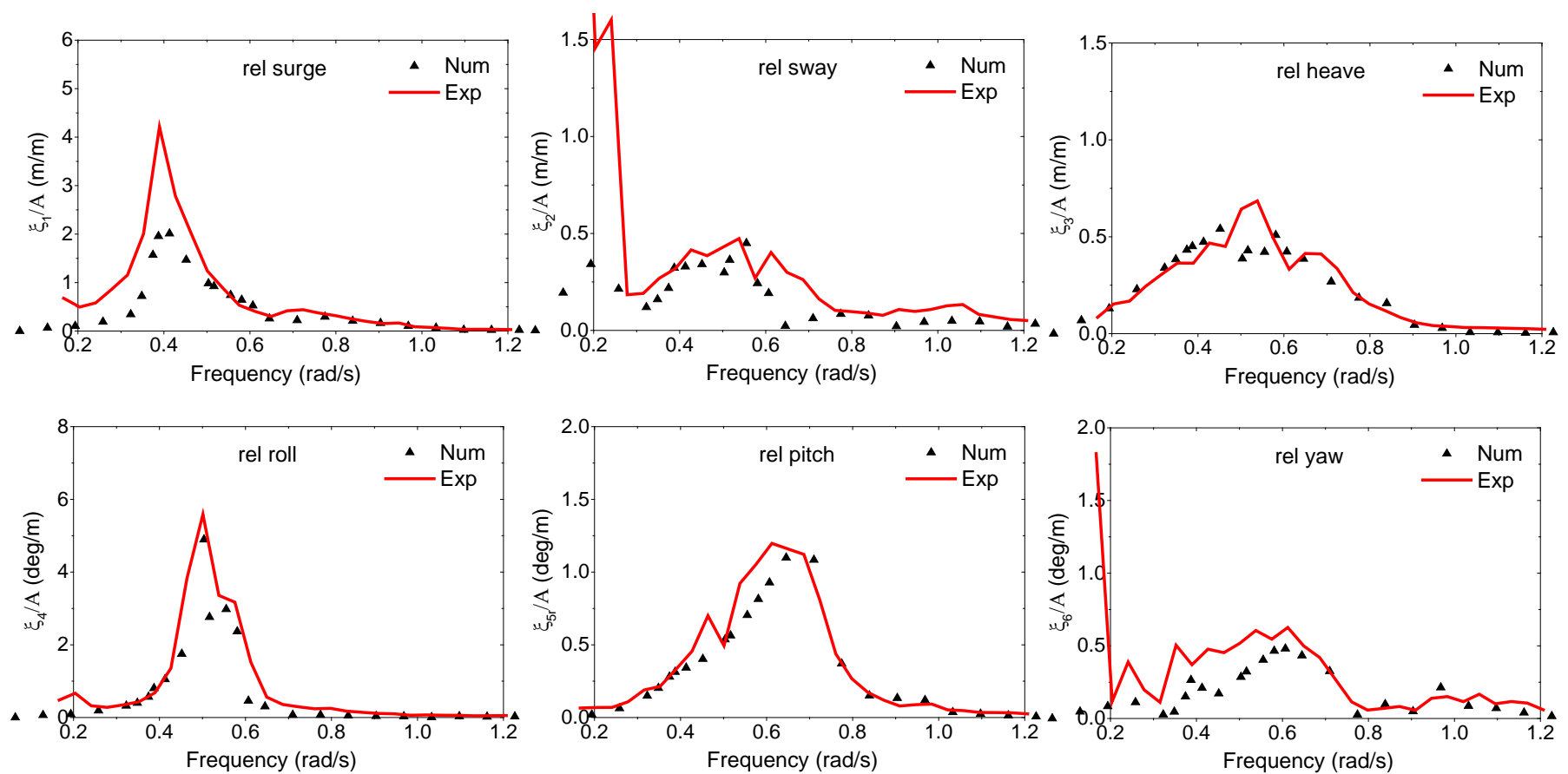

Fig. 32 RAOs of relative motions between vessels 

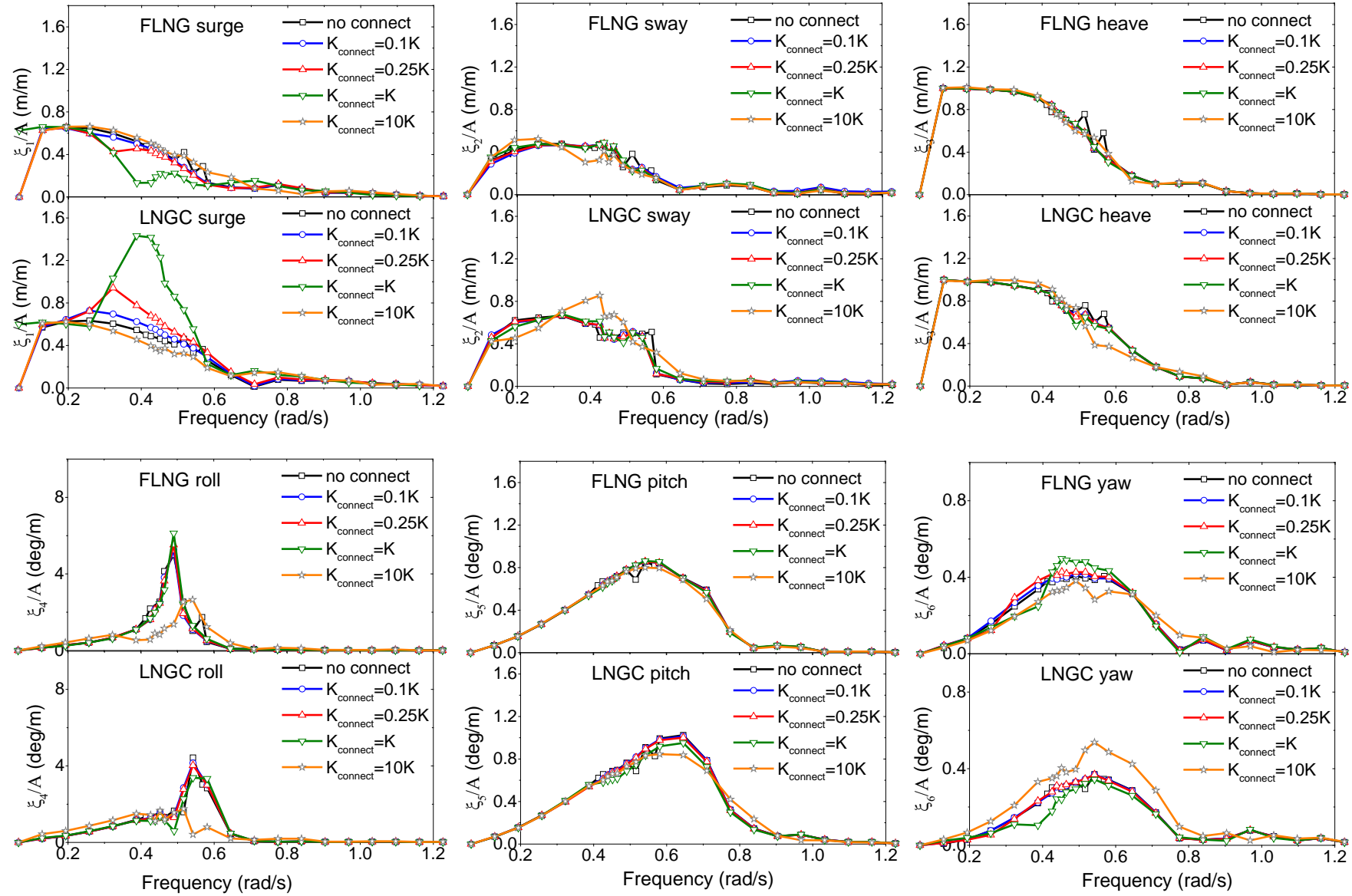

Fig. 33 Sensitivities of the vessels' motions to the stiffness of connection system
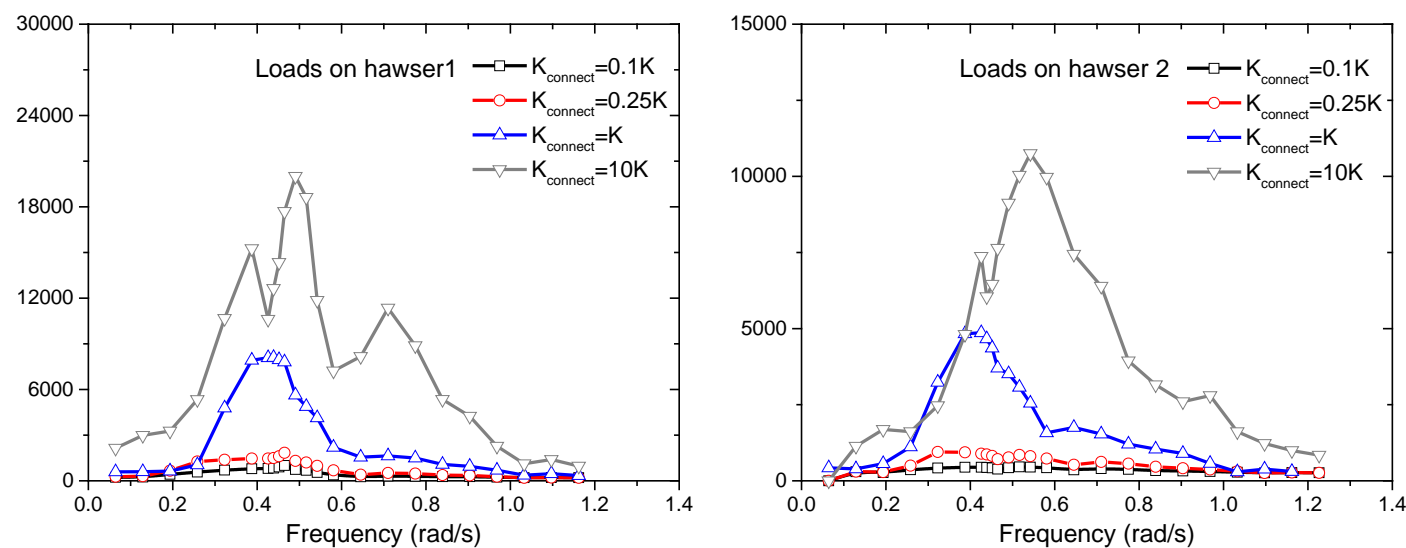

Fig. 34 Loads on hawsers for connection system with different stiffness 

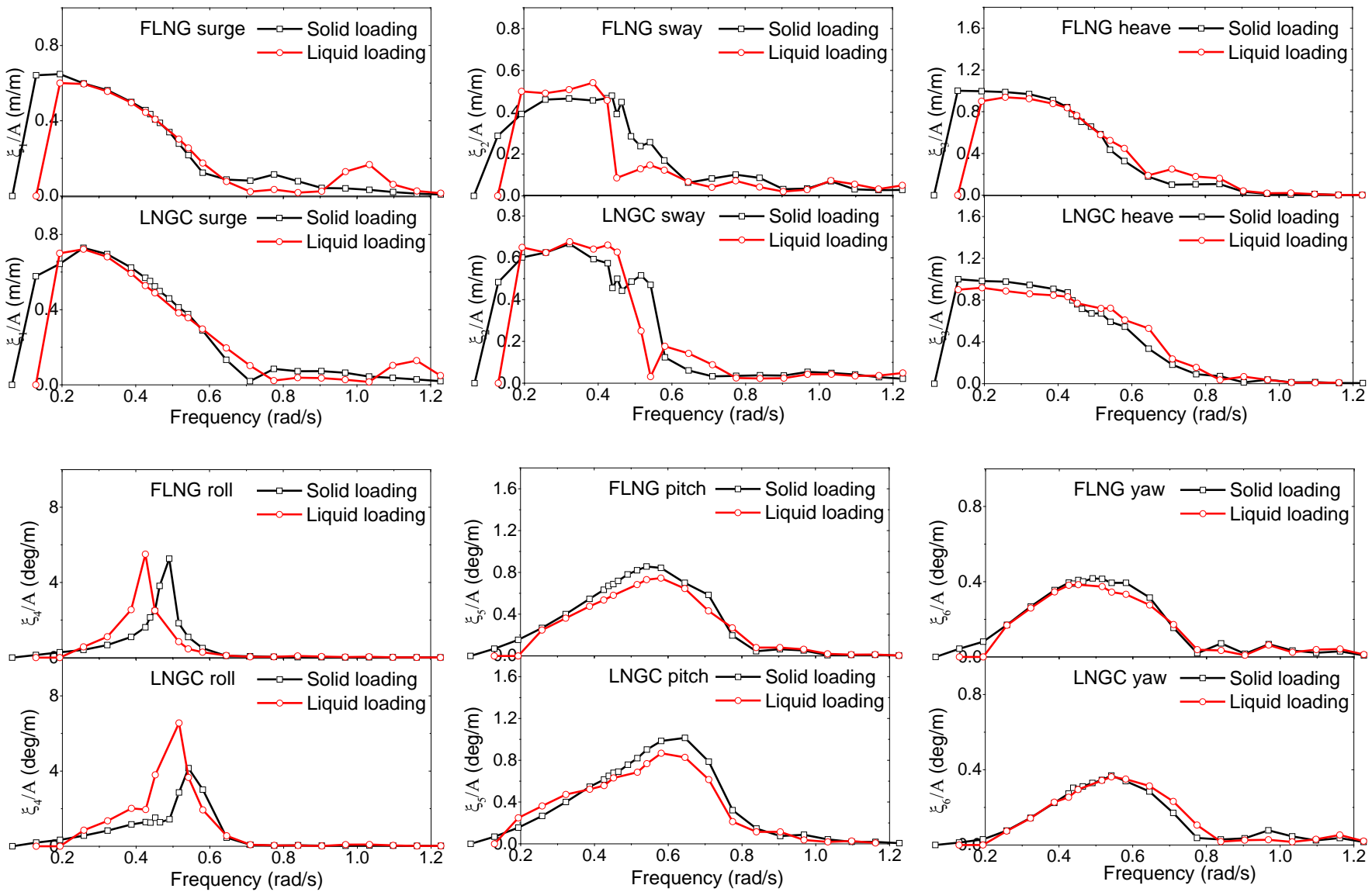

Fig. 35 RAOs of vessels' motions in solid and liquid loadings
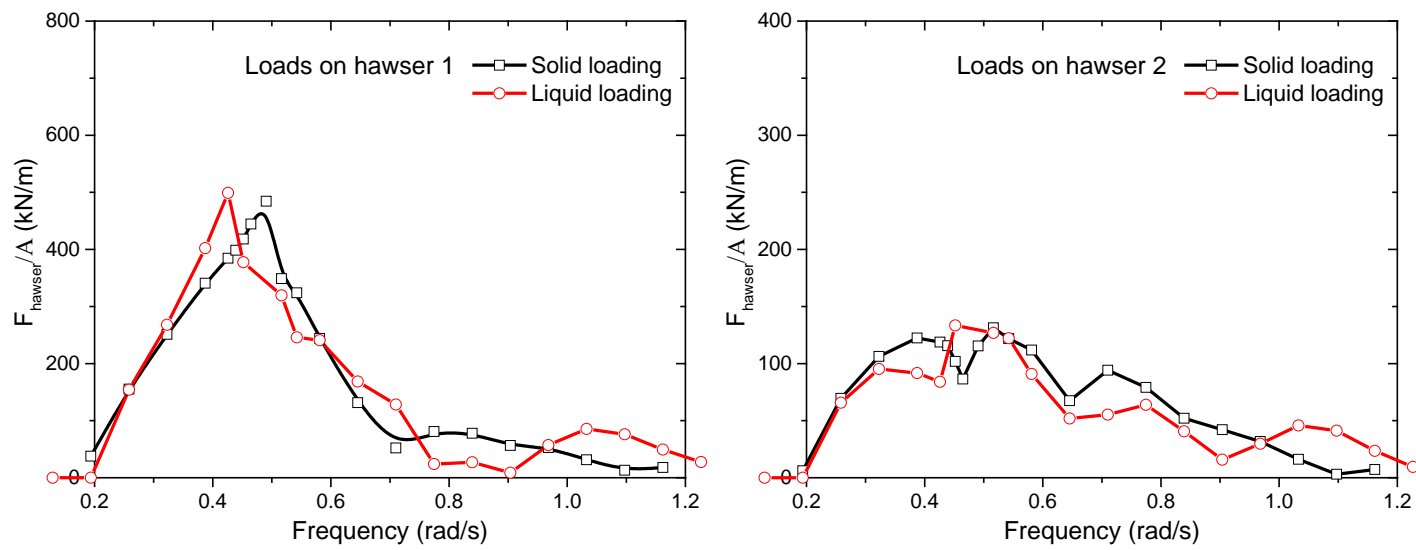

Fig. 36 Loads on hawsers for vessels in solid and liquid loadings

\section{Conclusion}

A high efficiency numerical code is developed to investigate the coupled responses of side-by-side arranged FLNG system. Experimental tests are conducted to validate the numerical code and provide more perceptual understanding of the coupling mechanism. Based on numerical and experimental studies, the hydrodynamic interactions between vessels, sloshing effects on vessels' motions, and responses of the connection system are investigated. The following conclusions are drawn: 
1) Numerical program developed in this study based on potential flow is reliable and of feasible efficiency in the prediction of liquid loading FLNG system responses, the damping-lid applied in the gap zone can give good results in hydrodynamic coefficients calculation.

2) Hydrodynamic interactions between vessels are sensitive to wave directions for shielding effects, vessels will subject to larger motion responses when located in weather side. Increased motion responses can also be excited by the radiation wave of the adjacent vessel.

3) The sloshing peaks in liquid loading vessels under beam sea mainly excited by motions in the roll and sway modes, respectively. Sloshing effects on vessels' sway motion can increase in the low filling level for the natural sloshing frequencies are more close to vessels' main response frequency region. With the increase of wave amplitude, nonlinear sloshing becomes obvious near the natural sloshing frequency; while vessels' motions do not demonstrate obvious nonlinearity.

4) Resonant responses of vessels can be excited when the resonant frequency of connection system equals wave frequency. The vessels' motions can be reduced significantly when the stiffness of the connection system is rather large and interactions between the vessels increase correspondingly.

\section{Acknowledgement}

This work was supported by State Key Lab of Ocean Engineering Shanghai Jiao Tong University and financially supported by China National Scientific and Technology Major Project (Grant No. 2016ZX05028-006-009); all of these supports are gratefully acknowledged by the authors. 


\section{References}

Buchner, B., Van Dijk, A., \& De Wilde, J. (2001). Numerical multiple-body simulations of side-by-side mooring to an FPSO. In The Eleventh International Offshore and Polar Engineering Conference. International Society of Offshore and Polar Engineers.

Chen, X. B. (2005). Hydrodynamic analysis for offshore LNG terminals. In Proceedings of the 2nd International Workshop on Applied Offshore Hydrodynamics, Rio de Janeiro.

Chen, Z., Zong, Z., Li, H. T., \& Li, J. (2013). An investigation into the pressure on solid walls in 2D sloshing using SPH method. Ocean Engineering, 59, 129-141.

Choi, Y. R., \& Hong, S. Y. (2002). An analysis of hydrodynamic interaction of floating multi-body using higher-order boundary element method. In The Twelfth International Offshore and Polar Engineering Conference. International Society of Offshore and Polar Engineers.

Faltinsen, O. (1993). Sea loads on ships and offshore structures (Vol. 1). Cambridge university press.

Faltinsen, O. M. (1978). A numerical nonlinear method of sloshing in tanks with two-dimensional flow. Journal of Ship Research, 22(3).

Hong, S. Y., Kim, J. H., Cho, S. K., Choi, Y. R., \& Kim, Y. S. (2005). Numerical and experimental study on hydrodynamic interaction of side-by-side moored multiple vessels. Ocean Engineering, 32(7), 783-801.

Huijsmans, R. H. M., Pinkster, J. A., \& De Wilde, J. J. (2001). Diffraction and radiation of waves around side-by-side moored vessels. In The Eleventh International Offshore and Polar Engineering Conference. International Society of Offshore and Polar Engineers.

Huijsmans, R. H. M., Pinkster, J. A., \& De Wilde, J. J. (2001). Diffraction and radiation of waves around side-by-side moored vessels. In The Eleventh International Offshore and Polar Engineering Conference. International Society of Offshore and Polar Engineers.

Inoue, Y., \& Islam, M. R. (1999). Comparative study of numerical simulation and the experimental results for a parallely connected FPSO and LNG in waves. In The Ninth International Offshore and Polar Engineering Conference. International Society of Offshore and Polar Engineers.

Jiang, S. C., Teng, B., Bai, W., \& Gou, Y. (2015). Numerical simulation of coupling effect between ship motion and liquid sloshing under wave action. Ocean Engineering, 108, 140-154.

Kodan, N. (1984). The motions of adjacent floating structures in oblique waves. Journal of energy resources technology, 106(2), 199-205.

Koo, B. J., \& Kim, M. H. (2005). Hydrodynamic interactions and relative motions of two floating platforms with mooring lines in side-by-side offloading operation. Applied Ocean Research, 27(6), 292-310.

Lee, S. J., Kim, M. H., Lee, D. H., Kim, J. W., \& Kim, Y. H. (2007). The effects of LNG-tank sloshing on the global motions of LNG carriers. Ocean Engineering, 34(1), 10-20.

Lu, L., Teng, B., Sun, L., \& Chen, B. (2011). Modelling of multi-bodies in close proximity under water waves-Fluid forces on floating bodies. Ocean Engineering, 38(13), 1403-1416.

Malenica, S., Zalar, M., \& Chen, X. B. (2003). Dynamic coupling of seakeeping and sloshing. In 13th International Offshore and Polar Engineering Conference, ISOPE, Honolulu, HI, May (pp. 25-30).

Mitra, S., Wang, C. Z., Reddy, J. N., \& Khoo, B. C. (2012). A 3D fully coupled analysis of nonlinear sloshing and ship motion. Ocean Engineering, 39, 1-13.

Molin, B. (2001). On the piston and sloshing modes in moonpools. Journal of Fluid Mechanics, 430, 27-50.

Molin, B., Remy, F., Kimmoun, O., \& Stassen, Y. (2002). Experimental study of the wave propagation and decay in a channel through a rigid ice-sheet. Applied ocean research, 24(5), 247-260.

Nakayama, T., \& Washizu, K. (1981). The boundary element method applied to the analysis of two-dimensional nonlinear sloshing problems. International Journal for Numerical Methods in Engineering, 17(11), 1631-1646.

Nam, B. W., Kim, Y., Kim, D. W., \& Kim, Y. S. (2009). Experimental and numerical studies on ship motion responses coupled with sloshing in waves. Journal of Ship Research, 53(2), 68-82.

Newman, J. N. (2001). Wave effects on multiple bodies. Hydrodynamics in ship and ocean engineering, 3, 3-26.

Newman, J. N. (2005). Wave effects on vessels with internal tanks. In Proceedings of the 20th Workshop on Water Waves and Floating Bodies, Spitsbergen, Norway. 
Pessoa, J., Fonseca, N., \& Soares, C. G. (2015). Numerical study of the coupled motion responses in waves of side-by-side LNG floating systems. Applied Ocean Research, 51, 350-366.

Rognebakke, O. F., \& Faltinsen, O. M. (2003). Coupling of sloshing and ship motions. Journal of Ship Research, 47(3), $208-221$.

Sun, L., Taylor, R. E., \& Taylor, P. H. (2010). First-and second-order analysis of resonant waves between adjacent barges. Journal of Fluids and Structures, 26(6), 954-978.

Veritas, B. (2010). Hydrostar for experts user manual.

Wu, G. X., Ma, Q. W., \& Taylor, R. E. (1998). Numerical simulation of sloshing waves in a 3D tank based on a finite element method. Applied Ocean Research, 20(6), 337-355.

Xu, X., Yang, J. M., Li, X., \& Xu, L. Y. (2015). Time-domain simulation for coupled motions of three barges moored side-by-side in floatover operation. China Ocean Engineering, 29, 155-168.

Yao, C. B., \& Dong, W. C. (2015). Modeling of fluid resonance in-between two floating structures in close proximity. Journal of Zhejiang University SCIENCE A, 16(12), 987-1000.

Zhao, D. Y., Hu, Z. Q., \& Chen, G. (2015). An investigation on two dimensional nonlinear sloshing in rectangular tank. In ASME 2015 34th International Conference on Ocean, Offshore and Arctic Engineering (pp. V001T01A045-V001T01A045). American Society of Mechanical Engineers.

Zhao, D. Y., Hu, Z. Q., \& Chen, G. (2017 a). Experimental investigation on dynamic responses of flng connection system during side-by-side offloading operation. Ocean Engineering, 136, 283-293.

Zhao, D. Y., Hu, Z. Q., Chen, G., Lim, S., \& Wang, S. Q. (2017 b). Nonlinear sloshing in rectangular tanks under forced excitation. International Journal of Naval Architecture and Ocean Engineering.

Zhao, W. H., Wolgamot, H. A., Taylor, P. H., \& Taylor, R. E. (2017). Gap resonance and higher harmonics driven by focused transient wave groups. Journal of Fluid Mechanics, 812, 905-939.

Zhao, W. H., Yang, J. M., \& Hu, Z. Q. (2012). Hydrodynamic interaction between FLNG vessel and LNG carrier in side by side configuration. Journal of Hydrodynamics, Ser. B, 24(5), 648-657.

Zhao, W. H., Yang, J. M., Hu, Z. Q., \& Tao, L. B. (2014). Prediction of hydrodynamic performance of an FLNG system in side-by-side offloading operation. Journal of Fluids and Structures, 46, 89-110.

ZHU, H. R., ZHU, R. C., \& MIAO, G. P. (2008). A time domain investigation on the hydrodynamic resonance phenomena of 3-D multiple floating structures. Journal of Hydrodynamics, Ser. B, 20(5), 611-616. 